\title{
Zur Kenntnis der individuellen Schwankungen des Blutdruckes beim gesunden Menschen. ${ }^{1}$ \\ Von
}

Cand. med. A. Tavaststjerna.

(Aus dem physiologischen Institut der Universität Helsingfors.)

Die Entwickelung, welche die zur Messung des Blutdruckes beim Menschen vorgeschlagenen Methoden während der letzten Jahre gewonnen haben, hat zahlreiche Untersuchungen über die bei einer und derselben Person unter verschiedenen Umständen vorkommenden Variationen des Blutdruckes veranlaBt, wogegen die bei verschiedenen Individuen stattfindenden Variationen nur in einem verhältnismäBig geringen Grade berücksichtigt worden sind.

Dies ist ohne Zweifel wesentlich ron der Unsicherheit veranlaBt, die in bezug auf die mit den betreffenden Apparaten erhaltenen absoluten Druckwerte lange stattgefunden hat, denn es ist ja selbstrerständlich, $d a B$ nur wenn diese einigermaBen exakt sind, der Vergleich zwischen verschiedenen Individuen berechtigt ist, während andererseits Zahlen, die an und für sich selbst mit großen Fehlern behaftet sind, dennoch wertvolle Aufschlüsse über die bei einer und derselben Person erscheinenden Veränderungen des Drackes abgeben können, da hier der so wesentliche EinfluB der Weichteile (Hant, Muskeln) als konstant erachtet werden $m u B$, bei verschiedenen Individuen aber vielfachen Schwankungen unterliegt und daher nicht ohne weiteres eliminiert werden kann.

Unter den neueren Methoden zur Bestimmung des Blutdruckes beim Menschen ist wohl die von Riva-Rocci im Jahre 1896 beschriebene zurzeit am meisten benutzt. Sein Apparat besteht, wie bekannt, aus einer pneumatischen Gummimanschette, die in der Regel um den Oberarm angebracht wird; sie steht einerseits mit einem Gummigebläse, andererseits mit einem Manometer in Verbindung. Durch das Gebläse wird Luft in die Manschette so lange getrieben,

1 Der Redaktion am 15. November 1908 zugegangen. 
bis der Radialispuls gerade nicht mehr fühlbar ist; der dann rom Manometer angezeigte Druck stellt den Maximaldruck in der Arterie dar; auch kann man ron rornherein durch das Gebläse einen so groBen Druck geben, daß die Pulsationen ganz ausbleiben, und dann durch Offnen eines Ventiles die Luft langsam heraustreten lassen, bis der Radialispuls wieder fühlbar ist, und den dabei stattfindenden Druck am Manometer ablesen.

Wie v. Recklinghausen ${ }^{1}$ nachwies, werden indessen mittelst der von Riva-Rocci empfohlenen nur 5 bis $6 \mathrm{~cm}$ breiten Manschette zu hohe Zahlen für den arteriellen Druck erhalten und zwar ist der Unterschied keineswegs konstant, sondern von der Dicke des Armes wesentlich abhängig. Er schlug daher vor, eine Manschette von $15 \mathrm{~cm}$ Breite zu benutzen, welche, wie der Autor sowohl theoretisch als experimentell nachweist, den an einer manometrischen Methode zu stellenden Anforderungen in hohem Grade genügte.

Trotzdem wurde die schmale Manschette, ror allem auf Grund von Sahlis Ausführungen, bis in der letzten Zeit vielfach benutzt. Durch 0. Müller and Blauels ${ }^{2}$ Versuche ist indessen die Streitfrage, wie es scheint, als endgültig erledigt anzusehen. Bei Amputationen bestimmten diese Autoren den Blutdruck teils mittelst eines mit der Arterie direkt verbundenen Manometers, teils mit dem Apparat von Riva-Rooci und zwar sowohl mit der schmalen als mit der breiten Manschette. Wie aus folgender Zusammenstellung hervorgeht, zeigen die mit der letzteren gewonnenen Zahlen eine sehr gute Übereinstimmung mit den wirklichen Druckwerten, während die schmale Manschette gar zu hohe Zahlen gegeben hat.

\begin{tabular}{|c|c|c|c|c|}
\hline $\begin{array}{l}\text { Ver- } \\
\text { such. }\end{array}$ & $\begin{array}{c}\text { Federmanometer; } \\
\text { Druckmaximum } \\
\text { wăhrend des Ro- } \\
\text { spirationsmaxi- } \\
\text { nums; mm Hg. }\end{array}$ & $\begin{array}{l}\text { Die breite } \\
\text { Manschette; } \\
\text { mm Hg. }\end{array}$ & $\begin{array}{c}\text { Die schmale } \\
\text { Manschette; } \\
\text { mm Hg. }\end{array}$ & Anmerkungen. \\
\hline I & $\begin{array}{l}121 \\
126\end{array}$ & $\begin{array}{l}130 \\
-\end{array}$ & $173-174$ & $\begin{array}{l}\text { Federmanometer in A. ra- } \\
\text { dialis, Manschette am } \\
\text { gleichseitigen Oberarm. }\end{array}$ \\
\hline II & $\begin{array}{l}120 \\
120\end{array}$ & $\begin{array}{c}130 \\
-\end{array}$ & $\frac{-}{170}$ & Desgleichen. \\
\hline III & $\begin{array}{l}109 \\
109\end{array}$ & $\begin{array}{c}116-118 \\
-\end{array}$ & $\frac{-}{129-130}$ & $\begin{array}{l}\text { Federmanometer in A. } \\
\text { brachialis, Manschetteam } \\
\text { gekreuzten Oberarm. }\end{array}$ \\
\hline
\end{tabular}

1 v. Recklinghausen, Archiv f. exper. Pathologie u. Pharmakol. 1901. Bd. XLVI. S. 86, 87, 110.

: O. Müller u. Bla u el, Deutsch. Arch. f. klin. Med. 1907. Bd. IXC. S.517. 
In den Versuchen I und II war der Umfang des Armes normal; betreffend Versuch III wird angegeben, daB hier die Weichteile des Armes "sehr dürftige" waren.

Nach diesen Bestimmungen würde also die breite Manschette Druckwerte geben, die um etwa 7.5 bis 8.3 Proz. die tatsächlich vorhandenen übersteigen, während die mit der schmalen Manschette gewonnenen, je nach der Dicke des Armes, mit +41 bis 19 Proz. von den wirklichen Druckwerten abweichen würden.

Dies alles gilt natürlich nur unter der Voraussetzung, daB die untereinander verglichenen Druckwerte an und für sich gleich groB waren und führt uns zunächst zu der Frage, was wir eigentlich mit dem Rira-Roccischen Apparate messen. Im AnschluB an Versuche von Bing ${ }^{1}$ bemerken 0. Müller und Blauel (a. a. 0.) in dieser Hinsicht folgendes. Bing fand, daß man durch Erwärmung bzw. Abkühlung eines Unterarmes verschiedene Druckwerte in beiden Brachiales erzielen kann, während der Aortadruck dabei keine Veränderung erleidet. Also muB diese Differenz davon abhängig sein, daß hier der Seitendruck in der A. brachialis gemessen worden ist. Durch die komprimierende Manschette wird die Seitenwand der A. brachialis belastet. Das Blut strömt zunächst noch ungehindert durch das GefäB, und der in ihm herrschende, der komprimierenden Manschette Widerpart leistende Druck ist die Resultante aus dem Seitendruck in der Subclaria und dem jeweiligen Widerstande in den peripher gelegenen Gefäßen. Wächst nun der auf die Seitenwand der Brachialis mittelst der Manschette ausgeūbte Druck weiter an, so kommt ein Punkt, bei welchem der aus den genannten Komponenten resultierende Druck im GefäB eben erreicht und schlieBlich überwunden wird. An diesem Punkte klappt das GefäB zusammen, und die bisher wenigstens während der Systole erhaltene Kontinuität des Blutstromes wird eben unterbrochen. In diesem Moment des Zusammenklappens der Arterie, des Verschwindens des Radialispulses, wird also gerade der Seitendruck der Brachialis überschritten. Bleibt das Gefäß nun verschlossen, so herrscht, da die peripheren Gebiete abgesperrt sind, ohne jeden Zweifel oberbalb der komprimierenden Manschette der reine Seitendruck der Subclavia. Unter diesem bahnt sich das Blut seinen Weg durch die sich wieder eröffnende Manschette. Sobald dann freilich der erste Pulsschlag in der Radialis fühlbar wird, ist die Kontinuitāt des Blutstromes mit den peripher gelegenen GefäBteilen wieder hergestellt, und der in diesem Augenblicke im GefäB vorhandene und an der

${ }^{1}$ Bing, Berliner klin. Wochenschrift. 1906. Nr. 52. S. 1650. 
Manschette abgelesene Druck resultiert schon wieder aus den beiden Einflüssen, dem zentralen und peripheren. Man bestimmt also offenbar zur Zeit des ersten Wiederauftretens des Pulses in der Radialis den Seitendruck in der Brachialis, zur Zeit des Verschwindens des Radialispulses den Seitendruck in der Subclavia. Beide Werte unterscheiden sich aber unter gewöhnlichen Verhältnissen nur wenig voneinander.

Seinerseits mißt das mit der Radialis endständig verbundene Mano. meter den Seitendruck in der Brachialis. Da nun die Messung nach Riva-Rocci-Recklinghausen sich teils auf diesen, teils auf den Seitendruck in der Subclavia bezieht, und zwischen letzteren nur ein geringes Gefälle herrscht, dürfte im großen und ganzen ein direkter Vergleich zwischen den beiden also erhaltenen Druckwerten - dem mit dem Federmanometer und dem mit der Manschette gewonnenen gestattet sein. Also wäre der oben erwähnte Unterschied von etwa 7 bis 8 Proz. als tatsächlich vorhanden aufzufassen. Da sich indessen eine vollständige Übereinstimmung der beiden Druckwerte nicht erwarten läßt, sind wir keineswegs dazu berechtigt, an den mittelst der Manschette bestimmten Zahlen eine bestimmte Korrektur anzubringen, und dies um so weniger, als die Zahl der vergleichenden Versuche nur sehr gering ist.

Unter Anwendung der Kompressionsmethode hat man auch versucht, den Minimaldruck in den Arterien zu bestimmen, um solcherart Zahlen für den mittleren Blutdruck zu bekommen. Da sich meine eigenen Untersuchungen indessen allein auf den maximalen Blutdruck beziehen, habe ich keine Veranlassung, die Arbeiten über den Minimaldruck hier zu besprechen.

Ich will nur bemerken, daB die Bestimmung des IIaximaldruckes allein keinen vollständig genūgenden AufschluB über den Blutdruck geben kann.

Bei meinen Bestimmungen benutzte ich die breite Manschette nach v. Recklinghausen. Sie wurde am Oberarm befestigt und der Druck an einem Hg-Manometer abgelesen. Durch Palpation der A. radialis wurde sowohl das Verschwinden wie das Wiedererscheinen des Pulses beobachtet und das Mittel aus diesen Bestimmungen notiert. Nach meinen Erfahrungen läBt sich das Wiedererscheinen des Pulses viel schärfer als das Verschwinden feststellen. In der Regel wurden eine bis zwei Kontrollmessungen vorgenommen.

Folgende, gröBtenteils schon v. Recklinghausen und Hensen angegebenen VorsichtsmaBregeln wurden bei diesen Bestimmungen genau beobachtet:

Die Manschette und wenn möglich der ganze Arm muB in der 
Höhe des unteren Teiles des Brustbeines (Angulus Ludovici) gehalten werden;

die Manschette soll den Arm dicht umschließen, jedoch ohne zu strammen; wenn sie zu locker befestigt ist, so wirkt sie wie eine schmälere Manschette; ist sie dagegen zu stramm angebracht, wird eine Blutstaunng hervorgerufen, die unter anderem die Palpation des Radialispulses erschwert;

die Messung soll möglichst schnell stattfinden, weil bei länger dauernden Kompression des Armes eine Drucksteigerung eintritt;

bei wiederholten Messungen soll die Quecksilbersäule nach jeder Messung auf Null herabsinken;

die Armmuskeln sollen vollständig schlaff sein.

Bevor ich meine eigenen Resultate bespreche, ist es angezeigt, die Erfahrungen über die bei einer und derselben Person erscheinenden Variationen des Blutdruckes kurz zu erwähnen, um Gesichtspunkte für die Frörterung meiner eigenen Beobachtungen zu bekommen.

Da der Blutdruck, so viel es sich zurzeit übersehen läBt, bei verschiedenen Individuen auf verschiedene äuBere und innere Einflüsse sehr verschieden reagiert, ist es natürlich, $\mathrm{daB}$ die Angaben verschiedener Autoren große Differenzen darbieten und in einzelnen Fällen sogar einander direkt widersprechen. Hierzu hat übrigens auch der Mangel bei den zu diesem Zwecke benutzten Untersuchungsmethoden nicht unwesentlich beigetragen. Schon der Umstand, daB wir einen mehr oder minder peripheren Druck messen, dessen Schwankungen keineswegs immer mit denjenigen in der Aortawurzel ûbereinstimmen müssen, ist unzweifelhaft nicht ohne schädlichen EinfluB gewesen.

Aus den Untersuchungen von Gumprecht ${ }^{1}$, Hensen ${ }^{2}$, v. Recklinghausen ${ }^{3}$ und anderen geht hervor, daB sich der Blutdruck beim Menschen oft, ohne daB man die Ursache davon nachweisen kann, verändert. Im allgemeinen sind die Druckschwankungen aber ziemlich klein, können indessen innerhalb kürzester Zeit 10 bis $15 \mathrm{~mm} \mathrm{Hg}$ betragen, im Laufe einer Stunde den Betrag von $25 \mathrm{~mm} \mathrm{Hg}$ and im Laufe von 24 Stunden einen von 40 bis $60 \mathrm{~mm}$ Hg erreichen. Andererseits ist zu bemerken, daB der Druck bei einer und derselben Person gewissermaßen bestrebt ist, sich auf die Dauer an einem gewissen

1 Gumprecht, Zeitschr. f. klin. Med. 1900. Bd. XXXIX. S. 387 bis 391.

2 Hensen, Deutsches Arch. f. klin. Med. Bd. LXVII. S. 461 bis 467.

s. Recklinghausen, a. a. O. S. 123 bis 127. 
Nireau zu erhalten. Wenn man also mehreremal täglich während mehreren Tagen den Druck bestimmt, so stimmen die an den einzelnen Tagen gewonnenen Durchschnittszahlen aufs nächste miteinander überein. Die Abweichung betrug bei Hensen etwa $10^{\mathrm{mm}} \mathrm{Hg}$, bei Gumprecht indessen oft etwa $20 \mathrm{~mm}$ und mebr.

Inwiefern eine bestimmte Abhängigkeit zwischen den Tageszeiten und dem Blutdrucke stattfindet, ist noch nicht entschieden. Hensen fand den Druck im allgemeinen etwas (5 bis $15 \mathrm{~mm} H \mathrm{Hg}$ ) höher am Abend als am Morgen; von dieser Regel kamen indessen viele Ausnahmen vor. Auch Zadek ${ }^{1}$ beobachtete in mehreren Fällen nachmittags eine Zunahme des Druckes, die jedoch von der Mittagsmahlzeit unabhängig war; gegen Abend sank der Druck in den meisten Fällen herab. Andererseits werden diese Tagesschwankungen von Kluge ${ }^{2}$ bestritten und aus Jellineks ${ }^{3}$ umfassenden Versuchen scheint hervorzugehen, daB der Druck in der Regel frühmorgens und spätabends den niedrigsten Wert hat, und sein Maximum nachmittags nach der Hauptmahlzeit aufweist. Bei einem per Rektum ernährten Kranken konnte Hensen keine Tagesschwankungen des Druckes nachweisen. Bei Kranken, die auf eine. sehr mäBige Ernährung gesetzt werden mußten, fand Sommerfeld ${ }^{4}$ keine Druckvariationen zu verschiedenen Tageszeiten.

Angesichts dieser Erfahrungen kann man wohl kaum der Aufnahme von Nahrung oder der Verdauungsarbeit eine wesentlichere Bedeutung für diese Variationen zuschreiben.

Auch stellt Schüle ${ }^{5}$ die Einwirkung der Verdauungsarbeit ganz in Abrede; Colombo, WeiB ${ }^{6}$ und Recklinghausen beobachteten während der Verdauung eine Druckabnahme.

Nach Maximowitsch, Riedel ${ }^{7}$ und Karrenstein ${ }^{8}$ würde die Zufuhr gröBerer Flüssigkeitsmengen den Druck steigern.

Über die Einwirkung der körperlichen Arbeit liegen zahlreiche Erfahrungen vor, von welchen ich nur die allerwichtigsten hier berücksichtigen kann.

Eine angestrengte Muskelarbeit bewirkt unter normalen Verhält-

1 Zadek, Zeitschrift f. klin. Med. 1881. Bd. II. S. 521.

- Kluge, Die Messung des Blutdrucks am Menschen mit Hilfe des Baschschen Sphygmomanometers. Kieler Dissert. 1893.

S Jellinek, Zeitschrift f. klin. Med. 1900. Bd. XXXIX. S. 447.

- Sommerfeld, Therapeut. Monatshefte. 1901. S. 72.

- Schüle, Berliner klin. Wochenschrift. 1900. S. 736.

- Weiss, Münch. med. Wochenschrift. 1900. S. 69.

7 Riedel, zitiert nach Hensen.

B Karrenstein, Zeitschrift f. klin. Med. 1903. Bd. L. S. 322. 
nissen stets eine Drucksteigerung. Nach Ende der Arbeit sinkt der Druck schnell auf seine ursprüngliche Höhe, mit kleineren Variationen nach oben oder nach unten (Maseng). ${ }^{1}$ Bei geübten Individuen ist die Drucksteigerung kleiner. Es scheint, als ob der Druck in näherer Beziehung zu der Willensanstrengung als zu der Arbeitsgröße stehen würde. Eine schwere Arbeit, an welche die Versuchsperson gewöhnt ist, läßt den Druck in der Regel unverändert. Vergleiche in dieser Hinsicht auch die Arbeiten von Jellinek und Karrenstein.

Nach Hensen verändert sich der Druck nicht bei lange dauerndem Bettliegen, beim Aufstehen rom Bette, beim Aufhören einer Muskeltätigkeit, an die man gewöhnt gewesen ist. Dagegen bemerkt Gumprecht, daB der Druck bei Arbeitern, die nach vorhergehender schwerer Arbeit ins Krankenhaus aufgenommen wurden, im Laufe der ersten Woche merkbar herabsinkt.

Die psychischen Vorgänge sind in bezug auf ihre Einwirkung auf den Blutdruck unter normalen Verhältnissen meines Wissens nie genauer untersucht worden. $\mathrm{DaB}$ sie in dieser Hinsicht nicht ohne Bedeutung sein dürften, folgt indessen aus mehreren einzelnen Beobachtungen.

Einen großen Einfluß übt das Gefühlsleben aus. Schon geringere Affekte genügen, um Drucksteigerungen von 10 bis $20 \mathrm{~mm} \mathrm{Hg}$ hervorzurufen, und bei stärkeren Gemütsbewegungen sind Drucksteigerungen um 30 bis $40 \mathrm{~mm} H g$ beobachtet worden (Gumprecht).

Nach Hensen kommt bei Kranken an den Tagen der Aufnahme in und der Entlassung aus dem Krankenhaus in der Regel ein etwas erhöhter Blutdruck vor. Zwei Stunden nach dem Einschlafen sinkt der Druck um 20 bis $35 \mathrm{~mm}$ Hg (Pilz). ${ }^{2}$

Durch Hustenstöße können Drucksteigerungen um 20 bis 30 bis $50 \mathrm{~mm} \mathrm{Hg}$ erhalten werden (Gumprecht). Dagegen sinkt der Druck bei tiefer Inspiration um 5 bis $10 \mathrm{~mm} \mathrm{Hg}$.

Nicht selten zeigen sich Verschiedenheiten beim Drucke in den beiden Armen, und zwar ist der Druck meistens etwas höher im rechten Arme.

Ferner übt die Körperlage der Versuchsperson bei der Messung einen deutlichen Einflub aus. Beim Stehen ist der Druck im allgemeinen höher als beim Sitzen (v. Recklinghausen, Hensen). Dagegen macht es keinen deutlichen Unterschied, ob die Versuchsperson

'Maseng, Deutsches Arch. f. klin. Med. 1902. Bd. LXXIV. S. 253.

${ }^{2}$ Pilz, Wiener klin. Wochenschrift. 1900. S. 276. 
liegt oder sitzt (Hensen). Wird aber beim Liegen das Kopfkissen entfernt, so dab der Kopf eine horizontale Stellung annimmt, so sinkt der Druck merkbar (um etwa $15 \mathrm{~mm} H \mathrm{Hg}$ nach v. Recklinghausen).

Meine Untersuchungen beziehen sich auf etwa 500 Individuen von verschiedenem Alter und Geschlecht.

Für das Alter 7 bis 19 Jahre beträgt die Zahl der Untersuchten 246; die Versuchspersonen waren Schulkinder.

Für das Alter 22 bis 25 Jahre wurden die Bestimmungen an 180 Soldaten des früheren finnländischen Leibgarde-Bataillons ausgeführt.

Die Bestimmungen für das Mannesalter beziehen sich auf 47 Schutzleute und einige andere Personen.

Endlich habe ich an 14 Greisen am Armenhaus der Stadt Helsingfors Messungen ausführen dürfen.

Die Gesamtzahl meiner Versuchspersonen beträgt somit 487.

Die Versuchspersonen befanden sich immer in sitzender Stellung.

Um ganz zuverlässige Durchschnittszahlen für den Druck bei verschiedenem Alter zu bekommen, hätte ich, wegen der Variabilität des Druckes bei verschiedenen Individuen, bei gewissen Altersklassen eine größere Anzahl Versuchspersonen zul meiner Verfügung haben sollen. Ferner bereitet der Umstand, daB sich meine Versuchsindividuen zur Zeit der Versuche nicht alle unter vollständig gleichen äußeren Bedingungen befanden - wie z. B. daB die Messungen zu verschiedener Tageszeit stattfanden - dem Vergleich der einzelnen Bestimmungen gewisse Schwierigkeiten. Indessen wurden sämtliche Soldaten zu derselben Zeit, zwischen 8 Uhr 30 Minuten und 9 Uhr vormittags untersucht; sie hatten ihr Frühstück genossen und noch nicht ihre körperlichen Tbungen begonnen.

Die übrigen Versuchspersonen wurden gröBtenteils am Vormittage, zwischen Frühstück und Mittagsessen, in der Regel nach vorhergegangenen Studien oder leichter körperlicher Arbeit, beobachtet. Bei diesen würde man also, nach den oben Angeführten, einen etwas erhöhten Blutdruck zu erwarten haben.

Bei den Schulkindern wurde der Blutdruck während der Gymnastikstunde gemessen. Dabei wurde indessen immer beobachtet, daB die betreffenden Individuen wenigstens eine kürzere Zeit vor der Messung sich ruhig gehalten hatten. 
Zur Kennteis der individuellen Schwankungen usw. 413

Die psychische Einwirkung, die sich bei der Druckmessung nicht selten geltend macht und abnorme Druckwerte verursachen kann, versuchte ich dadurch zu vermeiden, dab ich insbesondere an den empfindlicheren Kindern die Bestimmungen mehreremal wiederholte, um sie solcher Art an diese zu gewöhnen und vollständig zu beruhigen. Die Angaben über die Pulsfrequenz zeigen, dab dies der Hauptsache nach mir gelungen ist.

Über den Blutdruck während des Säuglings- und des früheren Kindesalters habe ich keine eigenen Erfahrungen, weshalb ich mir gestatte, nach Hensen und Kook (Riva-Rocci, schmale Manschette), Anna Kolossowa ${ }^{1}$ und Shaw (Gärtners Tonometer), diese zu vervollständigen. Die betreffenden Angaben sind hauptsächlich Vierord ts Daten und Tabellen S. 242 (1907) entlehnt.

\begin{tabular}{|c|c|c|c|}
\hline Alter & $\begin{array}{c}\text { Zahl der } \\
\text { untersuchten } \\
\text { Individuen }\end{array}$ & $\begin{array}{c}\text { Maximaler } \\
\text { Blutdruck } \\
\text { mm Hg }\end{array}$ & Autor \\
\hline 1 Monat & - & $70-75$ & \\
\hline $1 / 2-1 . J a h r$ & - & $86-85$ & \\
\hline 2. & 一 & $80-90$ & Kook \\
\hline 3. " & - & $90-100$ & \\
\hline 3.-10. " , & — & $95-115$ & \\
\hline 4.-6. " & 8 & 107 & \\
\hline 11.-12. & 8 & 122 & Hensen, Mittel \\
\hline 1.-2. " " & & $80-85$ & \\
\hline 3.- 4. " & & 85 & \\
\hline 5.-7. " , & samt 340 & $90-95$ & Kolossowa, Mittel \\
\hline 8.-10. " " & & $95-100$ & \\
\hline 11. - 13. " & & $100-110$ & \\
\hline $1 / 4-12$. & 45 & $90-110$ & Shaw \\
\hline
\end{tabular}

Aus dieser Tabelle scheint hervorzugehen, daB der maximale Blutdruck während des Kindesalters allmählich ansteigt, je nachdem das Kind wächst und sich entwickelt.

Außer den Mittelwerten gibt Hensen auch die Grenzwerte an. Bei 15 gesunden Kindern im Alter von 4 bis 15 Jahren betrugen diese 1902.

1 Kolossowa, Pression sanguine chex les enfants. Thèse de Lausanne 
80 bzw. 135 mm Hg. Bei einem 11jährigen war der Druck sogar $150 \mathrm{~mm} \mathrm{Hg}$. Aus ihrem großen Beobachtungsmaterial reroffentlicht Kolossowa nur die Durchschnittszahlen. Über Kooks und Shaws Arbeiten besitze ich keine andere Kenntnis als die Angaben bei Vierordt.

So viel ich weiB, liegen keine ausführlichen Untersuchungen über den Blutdruck im Knaben- und Jünglingsulter vor, und meine Bestimmungen bezweckten daher in erster Linie, diese Lücke einigermaßen auszufüllen. Meine hierher gehörigen Resultate sind in den Tabellen I bis III eingetragen. Tabelle I bezieht sich auf den Blutdruck und die Pulsfrequenz bei den verschiedenen Altersklassen zwischen 7 und 19 Jahren ohne Berücksichtigung des Geschlechtes. In Tabelle II sind die Durchschnittszahlen gesondert für männliche und für weibliche Individuen angegeben. Endlich zeigt Tabelle III die individuelle Verteilung der Druckwerte bei den rerschiedenen Altersklassen ohne Berücksichtigung des Geschlechtes.

Tabelle I.

Maximaler Blutdruck und Pulsfrequenz im Alter von 7 bis 19 Jahren ohne Berücksichtigung des Geschlechts.

\begin{tabular}{|c|c|c|c|c|c|c|c|}
\hline \multirow{2}{*}{ 导 总 } & \multirow{2}{*}{ 岁骂 } & \multicolumn{3}{|c|}{$\begin{array}{c}\text { Blutdruck; } \\
\text { mm Hg }\end{array}$} & \multicolumn{3}{|c|}{$\begin{array}{c}\text { Pulsfrequenz } \\
\text { pro Minute }\end{array}$} \\
\hline & & Maximal & Minimal & Mittel & Maximal & Minimal & Mittel \\
\hline 7-9 & 12 & 96 & 72 & 87 & 108 & 72 & 91 \\
\hline 10 & 10 & 129 & 79 & 100 & 104 & 80 & 95 \\
\hline 11 & 16 & 120 & 82 & 102 & 108 & 64 & 87 \\
\hline 12 & 39 & 124 & 86 & 103 & 112 & 64 & 90 \\
\hline 13 & 39 & 126 & 84 & 107 & 112 & 64 & 89 \\
\hline 14 & 31 & 126 & 90 & 109 & 104 & 64 & 86 \\
\hline 15 & 27 & 133 & 94 & 114 & 104 & 68 & 83 \\
\hline 16 & 28 & 142 & 89 & 111 & 104 & 64 & 82 \\
\hline 17 & 21 & 138 & 100 & 112 & 88 & 60 & 79 \\
\hline 18 & 12 & 132 & 95 & 112 & 100 & 64 & 82 \\
\hline 19 & 11 & 140 & 103 & 121 & 96 & 64 & 79 \\
\hline
\end{tabular}


Zur Kenntwis der individuellen Schwankungen USW.

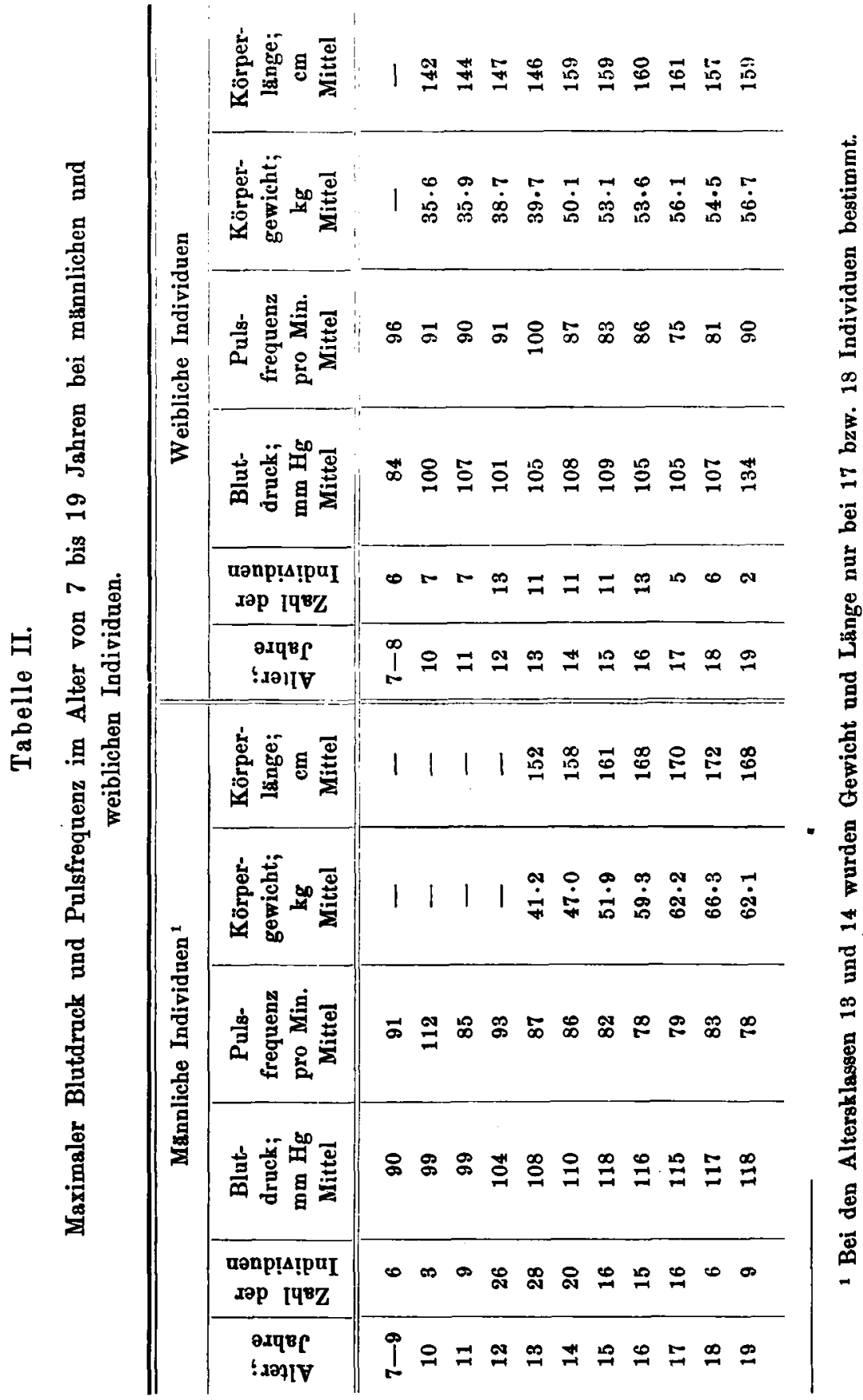


Aus der Tabelle I geht hervor, daB der Druck bis zum 15. Lebensjahre ununterbrochen ansteigt und dann während des 16 . bis 19 . Jahres etwa unverändert bleibt $(112 \mathrm{~mm} \mathrm{Hg}$ ). Im 20. Jahre begegnen wir einem wesentlich höberen Mittel, $121 \mathrm{~mm} \mathrm{Hg}$. Hier dürften indessen ver. schiedene Zufälligkeiten eine nicht unerhebliche Rolle gespielt haben. Erstens befanden sich unter den elf Versuchsindividuen nur zwei Frauen, und da diese, wie aus der Tabelle II ersichtlich, im allgemeinen einen

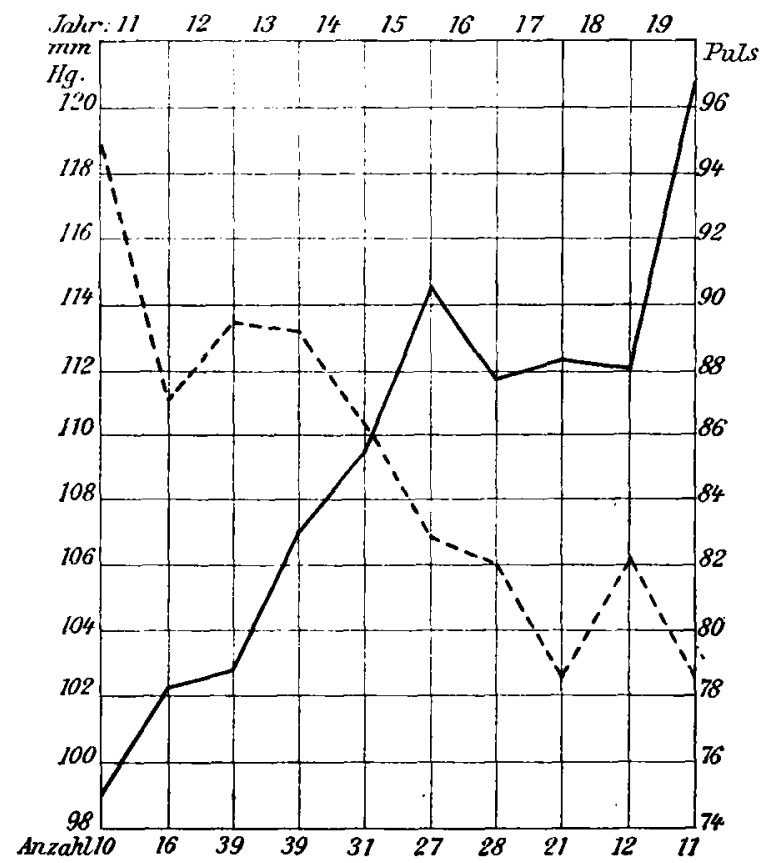

Fig. 1.

Maximaler Blutdruck $(\longrightarrow$ ) und Pulsfrequenz $(---)$ bei Individuen im Alter zwischen 10 und 19 Jahren, ohne Berücksichtigung des Geschlechtes.

niedrigeren Blutdruck haben, muB hier die Durchschnittszahl etwas größer als sonst werden. Es kommt aber hinzu, daB gerade die beiden za dieser Altersklasse gehörigen Frauen an und für sich einen abnorm hohen Druck von $134 \mathrm{~mm} \mathrm{Hg}$ hatten, was ebenso wie ihre hohe Pulsfrequenz wahrscheinlich von irgendwelcher zufalligen Indisposition bedingt war.

Aus der graphischen Darstellung der jetzt besprochenen Messungen in Fig. 1 folgt, daB der Druck vom 10. Lebensjahre an einen sehr regel- 
mäBigen Verlauf hat, nur mit der Ausnahme, daB im 16. Lebensjahre eine verhältnismäBig starke Steigerung erscheint. Es läßt sich ja nicht verneinen, dab hier eine Zufälligkeit vorliegt, andererseits zeigt aber Tabelle II, daB bei männlichen Indiriduen im 16. und 17. Lebensjahre, und bei weiblichen im 15. und 16. Lebensjahre die Druckwerte im Vergleich mit den nächst vorhergehenden und nachfolgenden etwas erhöht sind. Nun tritt gerade während dieser Lebensjahre die Pubertät in Finnland ein, wie u. a. aus Engströ $\mathrm{ms}^{1}$ Angaben hervorgeht, laut welchen die Menstruation hier am öftesten im Laufe des 15. und 16. Lebensjahres beginnt.

Es scheint mir nicht gerade unmöglich, daB der in den betreffenden Altersklassen beobachtete verhältnismäBig hohe Druck mit der Pubertätsentwicklung in einen gewissen Kausalzusammenhang gebracht werden könnte, indem z. B. der gerade zu dieser Zeit stattfindende stärkere körperliche Zuwachs hierbei von Bedeutung wäre. Auch könnten die in diesem Alter so oft vorhandenen nervösen Zustände einen EinfluB auf die Höhe des Blutdruckes ausüben. Ich muß indessen unbedingt zugeben, daB mein Beobachtungsmaterial doch viel zu wenig umfassend ist, um irgendwelche, einigermaBen sicher begründete Deutung zu gestatten.

Aus Tabelle II folgt ferner, daB der maximale Blutdruck während des sogen. Knaben-(Mädchen-)Alters, bis zum etwa 15. Lebensjahre bei beiden Geschlechtern etwa gleichgroB ist, vom 16. Lebensjahre an aber bei den männlichen Individuen schneller zunimmt als bei den weiblichen, so daB der maximale Druck bei den ersteren mit durchschnittlich $9 \mathrm{~mm} \mathrm{Hg}$ den bei den letzteren überragt.

Für die einzelnen Altersklassen (nach gefüllten Jahren) würde man also etwa folgende Durchschnittszahlen aufstellen können:

1. Knaben u. Mudehen 7 bis 9 Jahre

2. " " " 10,12 "

3. " " " $13,14 "$

4. Mädchen ...... 15,18 "

5. Jünglinge . . . 15,18 ,

\begin{tabular}{|c|c|c|c|c|}
\hline \multicolumn{5}{|c|}{$87^{\mathrm{mm}} \mathrm{Hg}-12$ Individuen. } \\
\hline & 103 & $"$, & -65 & $n$ \\
\hline 05 bis & 110 & $" n$ & -70 & " \\
\hline $105 \eta$ & 109 & $" n$ & -35 & $"$ \\
\hline $115 n$ & 118 & $"$ & - 53 & " \\
\hline
\end{tabular}

Der Druckwert für die erste Gruppe - $87^{\mathrm{mm}} \mathrm{Hg}$ - dürfte etwas zu klein sein, was zum Teil von der geringen Anzahl Beobachtungen herrührt.

Wie aus der Tabelle I hervorgeht, können das Maximum und das Minimum bei einer und derselben Altersklasse um sogar 40 bis $50 \mathrm{~mm}$ voneinander abweichen, und es ist daher lange nicht gestattet, auf Grund der soeben mitgeteilten Mittelwerte zu entscheiden, inwiefern ein vorhandener Druck als normal oder pathologisch aufzufassen ist.

1 Engs tröm, Finska Läkaresällskapets Handlingar. 1895. Bd.XLVI. S.222. 
Es empfiehlt sich daher, die stattgefundenen individuellen Variationen näher zu berücksichtigen, und gerade zu diesem Zwecke habe ich die Tabelle III entworfen.

Aus dieser ist ersichtlich, daB in jeder Alterklasse das Intervall der Druckwerte, die etwa ebenso oft vorkommen, ziemlich groB ist. Es ist daher nicht berechtigt, einen ganz bestimmten Druckwert als den normalen anzugeben. Vielmehr müssen als normal alle diejenigen Druckwerte angesehen werden, die innerhalb gewisser Grenzen fallen.

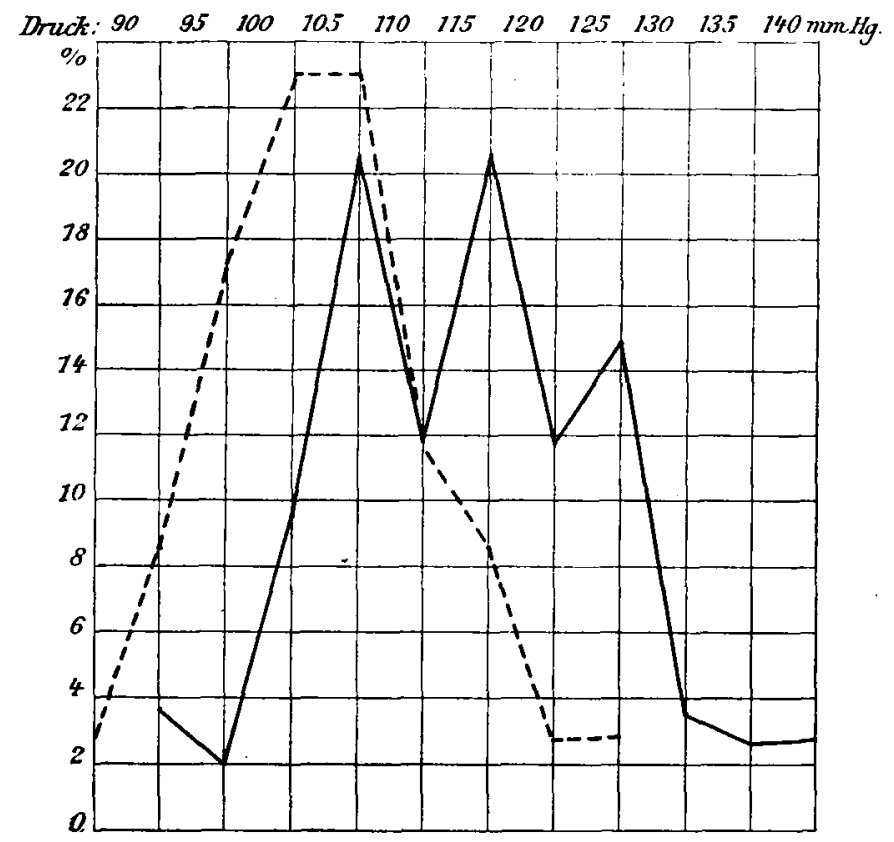

Fig. 2.

Die Verteilung der Druckwerte in die Gruppen 4 und 5. - Jünglinge, - - Mädchen.

Wenn wir als abnorm hoch oder niedrig. alle Druckwerte bezeichnen, die in der Tabelle III in weniger als 8 bis 10 Proz. vertreten sind, würden wir etwa folgende Zahlen für die verschiedenen Altersklassen aufstellen können:

1. Knaben und Mădchen 7 bis 9 Jahre 81 bis $100 \mathrm{~mm} \mathbf{H g}$.

2. " " $" 10,12$, $86,120 "$,

3. " " " 13,14 " $91,125, "$

4. Mädchen ...... 15,18 " 91,120 " "

5. Jünglinge ...... $15,18,100,130 "$, 
Bei allen diesen Altersklassen sind also Druckwerte um etwa $100 \mathrm{~mm} H \mathrm{Hg}$ als normal zu bezeichnen, und für die Gruppen 2 bis 5 gilt dasselbe von Druckwerten zwischen 100 und $120^{\mathrm{mm}} \mathrm{Hg}$ (vgl. auch Fig. 2).

Betreffend den Blutdruck im Alter der Reife besitze ich zahlreiche Messungen für männliche Individuen im Alter zwischen 22 und 25 Jahren. Die betreffenden Individuen waren wehrpflichtige Soldaten und das Material muB daher als so homogen wie möglich bezeichnet werden. In Tabelle IV sind Maximum, Minimum und Mittel des maximalen Druckes und der Pulsfrequenz bei diesen Individuen zusammengestellt. Tabelle $\nabla$ bringt eine prozentige Berechnung über die Verteilung der Druckwerte bei den einzelnen Individuen.

Tabelle IV.

Maximaler Blutdruck und Pulsfrequenz bei wehrpflichtigen Soldaten im Alter von 22 bis 25 Jahren.

\begin{tabular}{|c|c|c|c|c|c|c|c|}
\hline \multirow{2}{*}{ 这 } & \multirow{2}{*}{ 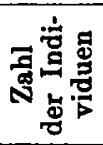 } & \multicolumn{3}{|c|}{$\begin{array}{c}\text { Maximaler Blutdruck; } \\
\text { mm Hg }\end{array}$} & \multicolumn{3}{|c|}{ Pulsfrequenz pro Minute } \\
\hline & & Maximum & Minimum & Mittel & Maximum & Minimum & Mittel \\
\hline $\begin{array}{l}22 \\
23 \\
24 \\
25\end{array}$ & $\begin{array}{l}58 \\
46 \\
56 \\
20\end{array}$ & $\begin{array}{l}142 \\
141 \\
152 \\
142\end{array}$ & $\begin{array}{r}92 \\
100 \\
95 \\
95\end{array}$ & $\begin{array}{l}116 \\
116 \\
117 \\
122\end{array}$ & $\begin{array}{r}100 \\
96 \\
92 \\
96\end{array}$ & $\begin{array}{l}48 \\
58 \\
56 \\
60\end{array}$ & $\begin{array}{l}75 \\
76 \\
73 \\
72\end{array}$ \\
\hline
\end{tabular}

Tabelle V.

Die individuellen Variationen des maximalen Blutdruckes bei wehrpflichtigen Soldaten im Alter von 22 bis 25 Jahren.

\begin{tabular}{|c|c|c|c|c|c|c|c|c|c|c|}
\hline \multirow{2}{*}{$\begin{array}{c}\text { Blutdruck; } \\
\text { mm Hg }\end{array}$} & \multicolumn{2}{|c|}{22 Jahre } & \multicolumn{2}{|c|}{23 Jahre } & \multicolumn{2}{|c|}{24 Jahre } & \multicolumn{2}{|c|}{25 Jahre } & \multicolumn{2}{|c|}{ Summa } \\
\hline & Zabl & $\%$ & Zabl & $\%$ & Zahl & $\%$ & Zahl & $\%$ & Zahl & $\%$ \\
\hline $91-95$ & 1 & $1 \cdot 7$ & 1 & $2 \cdot 2$ & 1 & 1.8 & 1 & 5 & 4 & $2 \cdot 2$ \\
\hline $96-100$ & 4 & $6 \cdot 9$ & 1 & $2 \cdot 2$ & 2 & $3 \cdot 6$ & - & - & 7 & $3 \cdot 9$ \\
\hline $101-105$ & 12 & $20 \cdot 7$ & 7 & $15 \cdot 2$ & 7 & $12 \cdot 5$ & 2 & 10 & 28 & $15 \cdot 6$ \\
\hline $106-110$ & 4 & 6.9 & 9 & $19 \cdot 6$ & 8 & $.14 \cdot 3$ & 2 & 10 & 23 & $12 \cdot 8$ \\
\hline $111-115$ & 10 & $17 \cdot 2$ & 4 & 8.7 & 9 & $16 \cdot 1$ & - & - & 23 & $12 \cdot 8$ \\
\hline $116-120$ & 8 & 13.8 & 7 & $15 \cdot 2$ & 10 & $17 \cdot 9$ & 4 & 20 & 29 & $16 \cdot 1$ \\
\hline $121-125$ & 8 & $13 \cdot 8$ & 9 & $19 \cdot 6$ & 5 & 8.9 & 2 & 10 & 24 & $13 \cdot 3$ \\
\hline $126-130$ & 4 & 6.9 & 4 & 8.7 & 7 & $12 \cdot 5$ & 4 & 20 & 19 & $10 \cdot 6$ \\
\hline $131-135$ & 5 & $8 \cdot 6$ & $\mathbf{3}$ & $6 \cdot 5$ & 3 & $5 \cdot 3$ & $\mathbf{3}$ & 15 & 14 & $7 \cdot 8$ \\
\hline $136-140$ & 1 & $1 \cdot 7$ & - & - & 1 & $1 \cdot 8$ & 1 & 5 & 3 & $1 \cdot 7$ \\
\hline $141-145$ & 1 & 1.7 & 1 & $2 \cdot 2$ & 1 & 1.8 & 1 & 5 & 4 & $2 \cdot 9$ \\
\hline-150 & - & - & - & - & 1 & 1.8 & - & - & 1 & 0.6 \\
\hline $151-155$ & - & 一 & - & - & 1 & 1.8 & - & - & 1 & 0.6 \\
\hline Sumtna & 58 & & 46 & & 56 & & 20 & & 180 & \\
\hline
\end{tabular}


Zur Kenntwis der individuellen Schwankungen usw. 421

Die in der Tabelle IV angenommenen Durchschnittszahlen zeigen vom 23. bis 26. Lebensjabre eine geringe, stetig fortschreitende $\mathrm{Zu}$ nalme von 116 bis auf $122 \mathrm{~mm} \mathrm{Hg}$, d. h. um etwa 5 Proz. Zu bemerken ist jedoch, da $B$ die Zahl der Untersuchten der letzten Gruppe nur 20 betrug.

Durchschnittlich ist der maximale Blutdruck bei den in der Tabelle IV aufgenommenen Individuen $117 \mathrm{~mm} \mathrm{Hg}$.

Der höchste ron mir beobachtete Druck beträgt $152 \mathrm{~mm}$, der niedrigste $90 \mathrm{~mm} \mathrm{Hg}$. Was die individuellen Variationen sonst betrifft, zeigt Tabelle V, daB der maximale Druck in den allermeisten

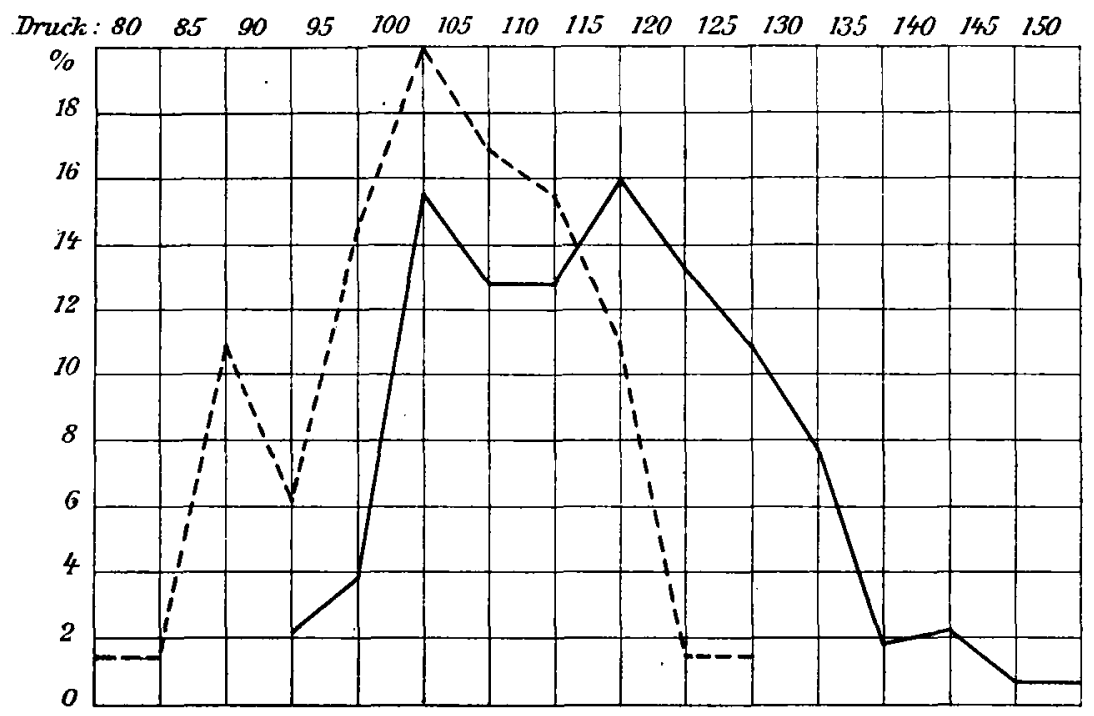

Fig. 3.

Die individuellen Variationen des maximalen Druckes bei Kìndern im Alter von 10 bis 12 Jahren (- - ) und bei Wehrpflichtigen im Alter von 22 bis 25 Jahren (-

Fällen zwischen 101 and $130 \mathrm{~mm}$ Hg schwankt, indem unter $180 \mathrm{In}$ dividuen 146, d. h. 81 Proz. einen Druck von dieser Höhe zeigten.

In Fig. 3 habe ich die individuellen Variationen bei sämtlichen Wehrpflichtigen mit denen bei der zahlreichsten ron mir untersuchten Gruppe von Schulkindern gefundenen zusammengestellt. Auch wenn zugegeben wird, daß, wegen der äußeren Umstände bei den Versuchen, die Druckwerte für die Kinder vielleicht etwas zu hoch ausgefallen sind, folgt jedenfalls aus dieser Zusammenstellung, daB einerseits bei 
Kindern in diesem Alter die gleichen Druckwerte wie bei Erwachsenen normal vorkommen, andererseits aber, daB die Kurve der Druckwerte bei den letzteren entschieden nach den höheren Zahlen hin verschoben ist. So finden wir bei den Wehrpflichtigen in keinem einzigen Falle einen Druck unter $90 \mathrm{~mm} \mathrm{Hg}$, und bei den Kindern in keinem einzigen Falle einen Druck über $130 \mathrm{~mm}$. Bei den Kindern beträgt der Druck in den meisten Fällen 95 bis $120 \mathrm{~mm}$, bei den Wehrpflichtigen 100 bis 130 usw.

Jellinek und Karrenstein, die früher den maximalen Blutdruck an einer großen Anzahl Soldaten bestimmten, bekamen dabei folgende Zahlen: Maximum 180, Mlinimum 80 (Jellinek, Anzahl untersuchter Individuen 532); Maximum 175, Minimum 75 (Karrenstein). Als normale Grenzwerte gelten nach Jellinek 100 bis $160 \mathrm{~mm}$, nach Karrenstein 101 bis $130 \mathrm{~mm} \mathrm{Hg}$; das Mittel beträgt beim letzteren 114 nm $\mathrm{Hg}$.

Wie ersichtlich, stimmen die Grenzwerte Karrensteins mit den meinigen genau überein; auch sein Mittel weicht nur um wenige Millimeter von meinem Mittel ab.

$\mathrm{DaB}$ die individuellen Variationen bei den Bestimumungen der soeben zitierten Autoren größer sind als bei den meinigen, erklärt sich wohl gröBtenteils daraus, $\mathrm{daB}$ ihre Druckmessungen mittels Gärtners Tonometer, also an einer peripheren Arterie, in welcher der Druck wahrscheinlich gröBere Schwankungen darbietet, stattfanden. Ich verkenne indessen nicht, daB die gröBere Anzahl der untersuchten Individuen hierhei einen gewissen EinfluB hat ausüben kōnnen.

Auch von der Altersklasse 26 bis 40 Jahre besitze ich einige Bestimmungen, die indessen nicht 80 zahlreich sind wie für die früheren. Diese beziehen sich auf Schutzleute, Unteroffiziere und einige Masseure, also auf Individuen mit etwa gleicher Beschäftigung wie die von mir untersuchten Wehrpflichtigen.

Die Resultate sind in der T'abelle VI zusammengestellt.

Tabelle VI.

Maximaler Blutdruck und Pulsfrequenz bei Schutzleuten, Unteroffizieren und Masseuren im Alter von 26 bis 45 Jahren.

\begin{tabular}{|c|c|c|c|c|c|c|c|}
\hline \multirow{2}{*}{$\begin{array}{l}\text { Alter, } \\
\text { Jahre }\end{array}$} & \multirow{2}{*}{$\begin{array}{c}\text { Zahl } \\
\text { der } \\
\text { Indi- } \\
\text { viduen }\end{array}$} & \multicolumn{3}{|c|}{ Maximaler Blutdruck; mm Hg } & \multicolumn{3}{|c|}{ Pulsfrequenz pro Minute } \\
\hline & & Maximum & Minimum & Mittel & Maximum & Minimum & Mittel \\
\hline $\begin{array}{l}26-30 \\
31-40 \\
41-45\end{array}$ & $\begin{array}{r}21 \\
21 \\
5\end{array}$ & $\begin{array}{l}142 \\
170 \\
180\end{array}$ & $\begin{array}{r}110 \\
99 \\
102\end{array}$ & $\begin{array}{l}121 \\
121 \\
134\end{array}$ & $\begin{array}{r}100 \\
100 \\
93\end{array}$ & $\begin{array}{l}56 \\
60 \\
74\end{array}$ & $\begin{array}{l}83 \\
81 \\
82\end{array}$ \\
\hline
\end{tabular}


Sowohl für die Altersklasse 26 bis 30 Jahre wie für die 31 bis 40 Jahre stimmt der mittlere Maximaldruck mit dem bei den 25 jährigen (Tabelle IV) genau überein. Hieraus würde folgen, daB sich der Druck von dem 25. bis zum 40. Lebensjahre etwa konstant erhält. Die Grenzwerte sind hier 170 , bzw. $99 \mathrm{~mm} \mathrm{Hg}$; das Maximum liegt also hier etwa $18 \mathrm{~mm}$ höher als bei den 25jährigen Wehrpflichtigen.

Bei fünf 40 bis 45 Jahre alten Schutzleuten wurde ein maximaler Druck ron durchschnittlich $134^{\mathrm{mm}} \mathrm{Hg}$ mit den Grenzwerten 180 und $102^{\mathrm{mm}}$ beobachtet. Die geringe Zahl der Beobachtungen gestattet indessen keine bestimmten Schlubfolgerungen daraus zu ziehen. Es wäre sehr wünschenswert umfangreichere Bestimmungen gerade für die Altersperiode 41 bis 50 Jahre zu besitzen, da während derselben die rïckgängigen Veränderungen allmählich beginnen, sich geltend $2 \mathfrak{u}$ machen, und insbesondere die ersten Stadien der Arteriosklerose erscheinen. Auch in der mir zugänglichen Literatur habe ich keine genaueren Angaben finden können.

Vom 51. Lebensjahre an liegen Beobachtungen von Ugroumoff mit dem v. Baschschen Sphygmomanometer und ron Masing mit Riva-Roccis Apparat und schmaler Manschette vor.

Masing ${ }^{1}$ bekam folgende Zahlen:

bei 19- bis 23 jährigen (9 Individuen) Maximum 164, Minimum 116, Mittel $141 \mathrm{~mm} \mathrm{Hg}$;

bei 38- bis 50jährigen (3 Individuen) Maximum 210, Minimum 114, Mittel $145 \mathrm{~mm}$ Hg;

bei 58- bis 81 jährigen (7 Individuen) Maximum 225, Minimum 114, Mittel $164^{\mathrm{mm}} \mathrm{Hg}$.

Ugroumoff bestimmte den Druck an den beiden Ellbogen, an den beiden FuBrücken, sowie an der Schläfe.

\begin{tabular}{c||c|c|c|r|r|r}
\hline $\begin{array}{c}\text { Alter, } \\
\text { Jahre }\end{array}$ & \multicolumn{2}{c|}{$\begin{array}{c}\text { Uruck am } \\
\text { Ellbogen }\end{array}$} & \multicolumn{2}{c|}{$\begin{array}{c}\text { Druck am } \\
\text { FuBrticken }\end{array}$} & \multicolumn{2}{c}{$\begin{array}{c}\text { Druck an der } \\
\text { Schlafe }\end{array}$} \\
\hline 51-60 & rechts & links & rechts & links & rechts & links \\
$61-70$ & 120 & 123 & 94 & 98 & 94 & 95 \\
$71-80$ & 141 & 142 & 109 & 103 & 105 & 104 \\
$81-96$ & 155 & 152 & 105 & 102 & 111 & 111 \\
& 147 & 143 & 107 & 106 & 109 & 111
\end{tabular}

Nach beiden Autoren ist der maximale Drack im höheren Alter, wenigstens bis zum 80 . Lebensjahre höher als im reifen, kräftigen Alter.

1 Masing, a. a. 0 . 
Ich hatte die Gelegenheit entsprechende Messungen an 14 männlichen Pfründern zu machen. Die einzelnen Beobachtungen sind in Tabelle VII zusammengestellt, die auch Angaben über den Zustand der A. radialis bringt.

Tabelle VII.

Maximaler Blutdruck und Pulsfrequenz bei Pfründern im Alter von 61 bis 91 Jahren.

\begin{tabular}{c|c|c|c||l}
\hline \hline Nummer & $\begin{array}{c}\text { Alter, } \\
\text { Jahre }\end{array}$ & $\begin{array}{c}\text { Maximaler } \\
\text { Blutdruck; } \\
\text { mm Hg }\end{array}$ & $\begin{array}{c}\text { Puls- } \\
\text { frequenz } \\
\text { pro Minute }\end{array}$ & \\
\hline \hline 1 & 61 & 175 & 64 & A. radialis \\
2 & 61 & 160 & 68 & keine deutliche Sklerose \\
3 & 66 & 130 & 58 & etwas Sklerose \\
4 & 72 & 155 & 72 & starke Sklerose \\
5 & 72 & 242 & 58 & \\
6 & 74 & 218 & 76 & \\
7 & 75 & 248 & 60 & \\
8 & 77 & 185 & 52 & etwas Sklerose \\
9 & 77 & 142 & 60 & \\
10 & 80 & 144 & 74 & keine deutliche Sklerose \\
11 & 83 & 155 & 90 & starke Sklerose \\
12 & 84 & 165 & 70 & keine deutliche Sklerose \\
13 & 90 & 140 & 70 & \\
14 & 91 & 190 & 80 & etwas Sklerose \\
starke Sklerose
\end{tabular}

Die für verschiedene Altersklassen berechneten Durchschnittszablen sind folgende:

Tabelle VIII.

Maximaler Blutdruck und Pulsfrequenz bei Pfründern im Alter von 61 bis 91 Jahren.

\begin{tabular}{c|c|c|c|c||c|c|c}
\hline \hline \multirow{2}{*}{$\begin{array}{c}\text { Alter, } \\
\text { Jahre }\end{array}$} & $\begin{array}{c}\text { Zahl } \\
\text { der } \\
\text { Indi- } \\
\text { viduen }\end{array}$ & \multicolumn{2}{|c|}{ Maximaler Blutdruck; mm Hg } & \multicolumn{2}{|c|}{ Pulsfrequenz pro Minute } \\
\cline { 3 - 7 } & Maximum & Minimum & Mittel & Maximum & Minimum & Mittel \\
\hline \hline $61-70$ & 3 & 175 & 130 & 155 & 68 & 58 & 63 \\
$71-80$ & 7 & 248 & 142 & 199 & 76 & 52 & 63 \\
$81-91$ & 4 & 190 & 140 & 169 & 90 & 70 & 79
\end{tabular}

Ich kann nicht hier, wie bei den früheren Tabellen, dafür garantieren, daB die Versuchsindividuen einer guten Gesundheit genossen. 
Bei den meisten dieser Greise war eine mehr oder minder ausgeprägte Arteriosklerose vorhanden, und auch in den Fällen, wo keine deutlichen Veränderungen in der A. radialis beobachtet werden konnten, lieBen sich krankhafte Störungen nicht bestimmt ausschließen. Bei einer 70jährigen gesunden Frau aus der wohlhabenden Klasse, dessen Radialis verhältnismäBig weich war, fand ich ennen Maximaldruck von $126 \mathrm{~mm} \mathrm{Hg}$.

Es fragt sich, inwiefern die bei diesen Greisen wie bei Greisen überhaupt beobachteten hohen Druckwerte dem in dem arteriellen System tatsächlich vorhandenen Druck entsprechen, oder ob der Kompressionswiderstand bei der mehr oder weniger starren GefäBwand hierbei in wesentlichem Grade beteiligt gewesen ist. Leider besitzen wir keine sicheren Gründe, um diese Frage zu beantworten. Nach v. Reck linghausen wäre der Widerstand der sklerotischen GefäBwand ohne irgendwelche wesentlichere Bedeutung und nach Hensens Berechnungen würde der Fehler bei der Bestimmung des Druckes höchstens etwa $20 \mathrm{~mm} \mathrm{Hg}$ betragen. In diesem Falle würde also der Druck bei den Greisen tatsāchlich in bedeutendem Grade den bei jüngeren Individuen übersteigen. Es $m a B$ indessen bemerkt werden, daB, auch wenn diese SchluBfolgerung richtig wäre, der mittlere Druck bei Greisen keineswegs in demselben $\mathrm{MaBe}$ wie der maximale erhöht ist, denn nach Strassburger ${ }^{1}$ ist der sogen. Pulsdruck (die Differenz zwischen dem systolischen und dem diastolischen Druck) bei Individuen mit starren Arterien verhältnismäßig groB. $\mathrm{Da}$ indessen die Arteriosklerose einen erhöhten Widerstand gegen die Blatströmung zuwege bringt, ist es nicht unmöglich, daB auch der mittlere Druck bei Greisen gesteigert ist.

Es liegt nahe $2 u$ denken, daB der im allgemeinen niedrigere Blutdruck bei Kindern im Vergleich mit dem bei Erwachsenen in dem 21. bis 40. Jahre zum Teil wenigstens von der geringeren KörpergröBe des Kindes bedingt ist, denn wie schon Hensen gefunden hat und meine mehr umfassenden Versuche dartun, übt das Körpergewicht einen, wenn auch geringen und inkonstanten EinfluB auf den Druck aus (vgl. unten). Daneben stellt sich aber die Frage, inwiefern das Alter an und für sich hierbei irgendwelche Bedeutung hat. Leider sind meine Aufzeichnungen des Körpergewichtes gerade für die Lebensjahre vor dem Pubertätsalter zu wenig zahlreich, um Resultate von größerer Tragweite zu gestatten. Die Drucksteigerung, der wir bei den Wehrpflichtigen begeguen, läBt uns indessen ein solches Verhalten ahnen, denn diese Steigerung kann nicht auf die verschiedene KörpergröBe

${ }^{1}$ Strassburger, Deutsches Archiv f. klin. Med. 1907. S. 378. 
bezogen werden, indem das Körpergewicht und die Körperlänge durchschnittlich bei den älteren Soldaten etwas kleiner gewesen sind als bei den jüngeren (vgl. Tabelle IX und XI). Wegen der großen Gleichförmigkeit, die ich in äußerer Beziehung bei den betreffenden Versuchen fand, und angesichts der rerhältnismäBig großen Zahl der untersuchten Individuen ist es doch nicht leicht, die erwähnte Erscheinung als eine reine Zufälligkeit aufzufassen.

Andererseits wissen wir aus neueren pathologisch-anatomischen Erfahrungen ${ }^{1}$, daß die Gefäßwand während der ganzen Wachstumsperiode bis in die Mitte oder das Ende des dritten Jahrzehntes an Dicke zunimmt, hauptsächlich auf Grund einer Zunahme der elastischen Elemente. Hierdurch wird natürlich die Dehnbarkeit der Gefảße vermindert, und daB dadurch der maximale Druck erhöht werden kann, folgt aus dem, was früher in bezug auf Greise hervorgehoben wurde.

Auch die Tatsache, daB der Blutdruck im Laufe der zunächst folgenden 15 Jahre keine weitere, deutlich ausgeprägte Steigerung erleidet, steht mit dem strukturellen Verhalten der Gefäßwand, wie es Aschoff dargestellt hat, in Ubereinstimmung, indem nach der Periode des Wachstums ein durchschnittlich nur bis in die Mitte oder das Ende des vierten Jahrzehnts dauernder Abschnitt folgt, in welchem die histologische Struktur so gut wie unverändert bleibt, um dann ganz allmählich mit wechselnder Schnelle in die dritte absteigende Periode der Gefäßveränderungen überzugehen.

Im AnschluB hierza möge noch hervorgehoben werden, daB die hier kurz dargestellten Anschauungen Aschoffs eine direkte Bestätigung durch die neueren Erfahrungen über die kubische Dehnbarkeit der Aorta (insbesondere diejenigen von Strassburger) gefunden haben.

Meine Bestimmungen gestatten noch die Frage nach den Beziehungen zwischen dem Körpergewicht, der Körperlänge usw. einerseits und dem maximalen Blutdruck andererseits im gewissen Umfange zu erörtern.

In bezug auf die Einwirkung des Körpergewichtes auf den Blatdruck beim Menschen gibt Hensen an, daB 13 Frauen, deren Körpergewicht weniger als $50 \mathrm{~kg}$ betrug, einen maximalen Blutdruck ron $128 \mathrm{~mm} \mathrm{Hg}$ zeigten, während zehn Frauen mit einem Gewicht über $60^{\mathrm{kg}}$ durchschnittlich einen maximalen Blutdruck von $140^{\mathrm{mm}} \mathrm{Hg}$ hatten.

1 Aschoff, Über Arteriosklerose und andere Sklerosen des GefäBsystems. Beiheft zur Medizinischen Klinik. 1908. 
Da Hensen indessen die schmale Manschette benutzte, bei deren Anwendung die Weichteile des Armes nicht ohne Bedeutung sind, liegt immer die Möglichkeit vor, daß der von ihm bei den schweren Individuen beobachtete höhere Druck von dem stärker entwickelten Fettpolster und Muskelgewebe d. h. von einem methodischen Fehler verursacht wāre.

\section{Tabelle IX.}

Korpergewicht und Blutdruck bei den in der Tabelle IV aufgenommenen Wehrpflichtigen.

\begin{tabular}{|c|c|c|c|c|c|}
\hline \multirow{2}{*}{$\begin{array}{l}\text { Alter, } \\
\text { Jahre }\end{array}$} & \multirow{2}{*}{$\begin{array}{c}\text { Zahl der } \\
\text { Indivi- } \\
\text { duen }\end{array}$} & \multirow{2}{*}{$\begin{array}{c}\text { Mittleres } \\
\text { Körper- } \\
\text { gewicht, kg }\end{array}$} & \multicolumn{2}{|c|}{$\begin{array}{c}\text { Maximaler Blutdruck, } \\
\text { mm Hg bei den }\end{array}$} & \multirow{2}{*}{ Differenz } \\
\hline & & & $\begin{array}{l}\text { schwereren } \\
\text { Individuen }\end{array}$ & $\begin{array}{l}\text { leichteren } \\
\text { Individuen }\end{array}$ & \\
\hline 22 & 54 & 73.8 & 120 & 109 & +11 \\
\hline 23 & 44 & $72 \cdot 8$ & 119 & 113 & +6 \\
\hline 24 & 56 & $71 \cdot 2$ & 123 & 117 & +6 \\
\hline 25 & 19 & 73.2 & 129 & 114 & +15 \\
\hline
\end{tabular}

Tabelle X.

Detaillierte Angaben über das gegenseitige Verhaltnis zwischen Körpergewicht nnd maximalem Blutdruck bei den in Tabelle IV aufgenommenen Wehrpflichtigen.

\begin{tabular}{|c|c|c|c|c|c|c|c|c|c|c|c|c|c|c|}
\hline \multirow[b]{2}{*}{$\begin{array}{c}\text { Körper- } \\
\text { gewicht } \\
\text { kg }\end{array}$} & \multicolumn{13}{|c|}{ Maximaler Blutdruck; $\mathbf{m m ~} \mathbf{H g}$} & \multirow[b]{2}{*}{$\begin{array}{l}\text { Zabl der } \\
\text { Indivi- } \\
\text { duen }\end{array}$} \\
\hline & $\frac{1}{1}$ & $\begin{array}{l}8 \\
7 \\
1 \\
8\end{array}$ & $\stackrel{20}{1}$ & $\stackrel{8}{7}$ & $\stackrel{10}{=}$ & $\begin{array}{l}\stackrel{8}{0} \\
-1 \\
0 \\
0 \\
-1 \\
\end{array}$ & 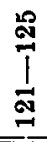 & 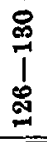 & $\stackrel{\substack{\infty \\
\hdashline}}{\mathfrak{\infty}}$ & 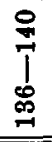 & $\frac{1}{7}$ & $\stackrel{0}{\dddot{0}}$ & $\stackrel{1}{9}$ & \\
\hline $91-92$ & & - & - & - & - & - & 1 & - & 一 & - & - & - & - & \\
\hline $89-90$ & - & - & - & - & 一 & - & - & 1 & - & - & - & - & 一 & 1 \\
\hline $87-88$ & - & 一 & 一 & - & 一 & - & 一 & - & - & - & - & - & - & - \\
\hline $85-86$ & - & - & 一 & 1 & 一 & - & - & 1 & 2 & - & - & - & - & 4 \\
\hline $83-84$ & - & - & 一 & - & 一 & - & - & 2 & - & - & - & - & - & 2 \\
\hline $81-82$ & 一 & - & 1 & 一 & 1 & - & 2 & 1 & 5 & - & 1 & - & 1 & 12 \\
\hline $79-80$ & - & - & 1 & 1 & - & 2 & 1 & 3 & - & 1 & - & - & - & 9 \\
\hline $77-78$ & - & 1 & - & 8 & $\mathbf{3}$ & $\mathbf{3}$ & 1 & - & 1 & 1 & 2 & - & 一 & 15 \\
\hline $75-7 \theta$ & - & - & 2 & - & 2 & 4 & 5 & 2 & 1 & $1-$ & 1 & - & - & 17 \\
\hline $73-74$ & - & $\mathbf{8}$ & 2 & 2 & 2 & 4 & 2 & 2 & 一 & - & - & 一 & - & 17 \\
\hline $71-72$ & 1 & 1 & 5 & 2 & 5 & 5 & $\mathbf{3}$ & 1 & 1 & - & - & - & 一 & 24 \\
\hline $69-70$ & 1 & - & 4 & 3 & 3 & 2 & 3 & 3 & 1 & - & - & 1 & - & 21 \\
\hline $67-68$ & - & - & 6 & 6 & 4 & 3 & 2 & 1 & 2 & 1 & - & - & - & 25 \\
\hline $65-66$ & $1-$ & 1 & $\mathbf{3}$ & 2 & 1 & 2 & 2 & 2 & - & - & - & 一 & - & 13 \\
\hline $63-64$ & 1 & 1 & 2 & 1 & 一 & - & - & - & 一 & - & - & - & - & 5 \\
\hline $61-62$ & 1 & - & 2 & 1 & - & 2 & - & - & - & - & - & - & - & 6 \\
\hline $59-60$ & - & - & 一 & - & - & - & - & - & - & - & - & 一 & - & - \\
\hline $57-58$ & $1-$ & - & - & - & 1 & - & - & - & - & - & - & - & - & 1 \\
\hline Summa & 4 & 7 & 28 & 22 & 22 & 27 & 22 & 19 & 13 & 3 & 4 & 1 & 1 & 173 \\
\hline
\end{tabular}


Meine mit der breiten Manschette ausgeführten Bestimmungen haben indessen das gleiche Resultat ergeben. Ich teilte die Wehrpflichtigen in zwei Gruppen ein, je nachdem ibr Körpergewicht kleiner oder gröBer war als das Mittel, und fand dabei, wie aus Tabelle IX ersichtlich, daß der mittlere Maximaldruck bei den schwereren Individuen etwa 6 bis 15 mı $\mathrm{Hg}$ höher war als bei den leichteren.

Indessen ist, wie aus der Tabelle $\mathrm{X}$ ersichtlich, das Verhältnis zwischen Körpergewicht und maximalem Blutdruck sehr inkonstant und der soeben hervorgehobene Sachverhalt tritt daher nur beim Vergleich der Durchschnittszahlen zum Vorschein. Hieraus folgt, daß wir nicht erwarten dürfen, bei schwereren Indiriduen in der Regel einem rerhältnismäBig hohen maximalen Blutdruck zu begegnen.

Die Körperlänge scheint auf den Blutdruck keinen deutlichen Einfluß auszuüben, insbesondere wenn die gleichzeitige Einwirkung des Körpergewichts ausgeschlossen ist. Vergleiche in dieser Hinsicht Tabelle XI und XII, welche keine nähere Erläuterung erfordern.

Tabelle XI.

Körperlange und Blutdruck bei den in der Tabelle IV aufgenommenen Wehrpflichtigen.

\begin{tabular}{|c|c|c|c|c|c|}
\hline \multirow{2}{*}{$\begin{array}{l}\text { Alter, } \\
\text { Jahre }\end{array}$} & \multirow{2}{*}{$\begin{array}{l}\text { Zabl der } \\
\text { Indivi- } \\
\text { duen }\end{array}$} & \multirow{2}{*}{$\begin{array}{c}\text { Mittlere } \\
\text { Körperlänge } \\
\text { cm }\end{array}$} & \multicolumn{2}{|c|}{$\begin{array}{c}\text { Maximaler Blutdruck, } \\
\text { mm } \mathrm{Hg}_{\mathrm{g}} \text { bei den }\end{array}$} & \multirow{2}{*}{$\begin{array}{c}\text { Differenz } \\
\mathrm{mm} \mathrm{Hg}\end{array}$} \\
\hline & & & $\begin{array}{c}\text { likngeren } \\
\text { Individuen } \\
\end{array}$ & $\begin{array}{c}\text { kürzeren } \\
\text { Individuen }\end{array}$ & \\
\hline $\begin{array}{l}22 \\
23 \\
24 \\
25\end{array}$ & $\begin{array}{l}54 \\
44 \\
56 \\
19\end{array}$ & $\begin{array}{l}174 \cdot 4 \\
173 \cdot 1 \\
171 \cdot 4 \\
172 \cdot 3\end{array}$ & $\begin{array}{l}117 \\
118 \\
122 \\
118\end{array}$ & $\begin{array}{l}114 \\
114 \\
113 \\
124\end{array}$ & $\begin{array}{l}+3 \\
+4 \\
+9 \\
-6\end{array}$ \\
\hline
\end{tabular}

Tabelle XII.

Körpergewicht, Körperlknge und maximaler Blutdruck bei den in Tabelle IV aufgenommenen Wehrpflichtigen.

\begin{tabular}{|c|c|c|c|c|c|}
\hline \multirow{2}{*}{$\begin{array}{l}\text { Körper- } \\
\text { gewicht } \\
\text { kg }\end{array}$} & \multirow{2}{*}{$\begin{array}{l}\text { Zahl der } \\
\text { Indivi- } \\
\text { duen }\end{array}$} & \multirow{2}{*}{$\begin{array}{c}\text { Mittlere } \\
\text { Körperlänge } \\
\text { cm }\end{array}$} & \multicolumn{2}{|c|}{$\begin{array}{c}\text { Maximaler Blutdruck, } \\
\text { mm Hg bei den }\end{array}$} & \multirow{2}{*}{$\begin{array}{c}\text { Differenz } \\
\text { mm } \mathbf{H g}\end{array}$} \\
\hline & & & $\begin{array}{l}\text { längeren } \\
\text { Individuen }\end{array}$ & $\begin{array}{l}\text { kürzeren } \\
\text { Individnen }\end{array}$ & \\
\hline $\begin{array}{l}65-66 \\
67-68 \\
69-70 \\
71-72 \\
73-74 \\
75-76 \\
77-78 \\
79-80\end{array}$ & \begin{tabular}{r|}
13 \\
25 \\
21 \\
24 \\
17 \\
17 \\
15 \\
9
\end{tabular} & $\begin{array}{l}168 \cdot 6 \\
171 \cdot 0 \\
171 \cdot 0 \\
172 \cdot 5 \\
172 \cdot 5 \\
173 \cdot 3 \\
176 \cdot 6 \\
176 \cdot 5\end{array}$ & $\begin{array}{l}113 \\
119 \\
113 \\
111 \\
110 \\
121 \\
116 \\
127\end{array}$ & $\begin{array}{l}113 \\
113 \\
118 \\
115 \\
116 \\
121 \\
112 \\
118\end{array}$ & $\begin{array}{l}- \\
+6 \\
-5 \\
-4 \\
-6 \\
-4 \\
+4 \\
+9\end{array}$ \\
\hline
\end{tabular}


Zur Kenntnis DER Individuellen Schwankungen usw. 429

Uber den EinfluB des Geschlechts bemerkt Hensen, daB er bei 25 Arbeitern im Alter von 17 his 30 Jahren einen durchschnittlich $5 \mathrm{~mm} \mathrm{Hg}$ höheren Druck als bei 30 Frauen desselben Alters beobachtete, faBt aber diesen Unterschied als Folgen des verschieden großen Körpergewichtes auf.

Bei den von mir untersuchten Individuen im Alter von 15 bis 18 Jahren war der Maximaldruck bei den Frauen etwa $9 \mathrm{~mm} \mathrm{Hg}$ niedriger als bei den Nännern.

Ob das Geschlecht an und für sich, also unabhängig vom Körpergewicht, hierbei irgendeinen Einflub ausübt, darüber lassen sich aus meinen Beobachtungen keine näheren Schlüsse ziehen. 19 Mädchen im Alter ron 16 bis 18 Jahren mit einem mittleren Körpergewicht von $55.4 \mathrm{~kg}$ hatten einen mittleren Maximaldruck von $106 \mathrm{~mm} \mathrm{Hg}$; 18 Jünglinge in demselben Alter mit einem mittleren Körpergewicht von $55 \cdot 3 \mathrm{~kg}$ zeigten einen mittleren Maximaldruck von $115 \mathrm{~mm} \mathrm{Hg}$. Wegen der geringen Anzahl der Beobachtungen und mit Hinsicht darauf, dab die Lebensjahre, die hier in Betracht kommen, der Pubertätsperiode zugehören, deren Einwirkung auf Jünglinge und Mädchen möglicherweise etwas verschieden ist, glaube ich, daB diesem Resultat keine weitere Bedeutung zuerkannt werden darf.

Schon von vornherein ist es nicht sehr wahrscheinlich, dab die Pulsfrequenz irgendeine deutlichere Einwirkung auf den Blutdruck bei Erwachsenen ausūben sollte. So ist auch der Fall, wie aus den Tabellen XIII und XIV ohne weitere Auslegungen herrorgeht.

\section{Tabelle XIII.}

Pulsfrequenz und maximsler Blutdruck bei den in der Tabelle IV aufgenommenen Wehrpflichtigen.

\begin{tabular}{c|c|c}
\hline $\begin{array}{c}\text { Pulsfrequenz } \\
\text { pro Minute }\end{array}$ & $\begin{array}{c}\text { Maximaler } \\
\text { Blutdruck } \\
\mathrm{mm} \mathrm{Hg}\end{array}$ & $\begin{array}{c}\text { Zahl der } \\
\text { Individuen }\end{array}$ \\
\hline \hline $56-60$ & 117 & 19 \\
$61-65$ & 118 & 14 \\
$66-70$ & 112 & 21 \\
$71-75$ & 114 & 34 \\
$76-80$ & 127 & 52 \\
$81-85$ & 116 & 21 \\
$86-90$ & 121 & 10
\end{tabular}


a. Tavaststjerna: Zur Kenntinis der Schwankongen usw.

\section{Tabelle XIV.}

Detaillierte Angaben über das gegenseitige Verbaltnis 2 wischen Pulsfrequenz und maximalem Blutdruck bei den in Tabelle IV aufgenommenen Wehrpflichtigen.

\begin{tabular}{|c|c|c|c|c|c|c|c|c|c|c|c|c|}
\hline \multirow[b]{2}{*}{$\begin{array}{l}\text { Blutdruck } \\
\mathrm{mm} \mathrm{Hg}\end{array}$} & \multicolumn{11}{|c|}{ Pulsfrequenz pro Minute } & \multirow[b]{2}{*}{$\begin{array}{c}\text { Zahl der } \\
\text { Indivi- } \\
\text { duen }\end{array}$} \\
\hline & 号 & $\frac{1}{10}$ & $\prod_{0}^{8}$ & $\frac{18}{10}$ & $\stackrel{1}{1}$ & $\overbrace{1}^{1}$ & $\begin{array}{l}\infty \\
1 \\
0 \\
0\end{array}$ & $\frac{\infty}{10}$ & $\stackrel{8}{1}$ & $\frac{10}{1}$ & $\begin{array}{l}8 \\
1 \\
\\
8\end{array}$ & \\
\hline $90-95$ & - & - & - & - & 2 & - & 1 & 1 & - & - & - & 4 \\
\hline $96-100$ & 1 & - & - & - & 2 & 1 & 1 & 2 & - & - & - & 7 \\
\hline $101-105$ & - & - & 4 & 1 & 3 & 6 & 8 & 4 & 2 & - & - & 28 \\
\hline $106-110$ & 1 & - & 3 & 3 & 2 & 1 & 11 & - & 1 & 1 & - & 23 \\
\hline $111-115$ & - & - & 2 & 3 & 2 & 4 & 8 & 3 & 1 & - & - & 23 \\
\hline $116-120$ & - & - & 3 & 1 & 6 & 5 & 6 & 5 & - & 1 & 1 & 28 \\
\hline $121-125$ & - & - & 5 & 3 & 2 & 4 & 6 & 3 & 2 & - & - & 25 \\
\hline $126-130$ & - & - & - & 2 & 1 & 5 & 6 & 1 & 2 & 1 & 1 & 1 \\
\hline $131-135$ & - & - & 1 & 1 & 1 & 6 & 2 & - & 1 & - & 2 & 14 \\
\hline $136-140$ & - & - & 1 & - & - & - & 2 & - & - & - & $-!$ & 3 \\
\hline $141-145$ & - & - & - & - & - & - & 1 & 2 & 1 & - & - & 4 \\
\hline $146-150$ & - & - & - & - & - & 1 & - & - & - & - & - & 1 \\
\hline $151-155$ & $=$ & $=$ & - & - & $=$ & 1 & - & - & - & - & - & 1 \\
\hline Summa & 2 & - & 19 & 14 & 21 & 34 & 52 & 21 & 10 & $\mathbf{3}$ & 4 & 180 \\
\hline
\end{tabular}

Nur bei wachsenden Kindern kann zwischen Pulsfrequenz und Blutdruck insofern eine bestimmte Beziehung nachgewiesen werden, daB der Druck bei zunehmendem Alter zunimmt, während die Pulsfrequenz gleichzeitig abnimmt (vgl. die graphische Darstellung in Fig. 1). $\mathrm{DaB}$ diese Erscheinungen indessen nicht als Ursache und Wirkung aufgefaßt werden dürfen, brauche ich wohl kaum hervorzuheben. 


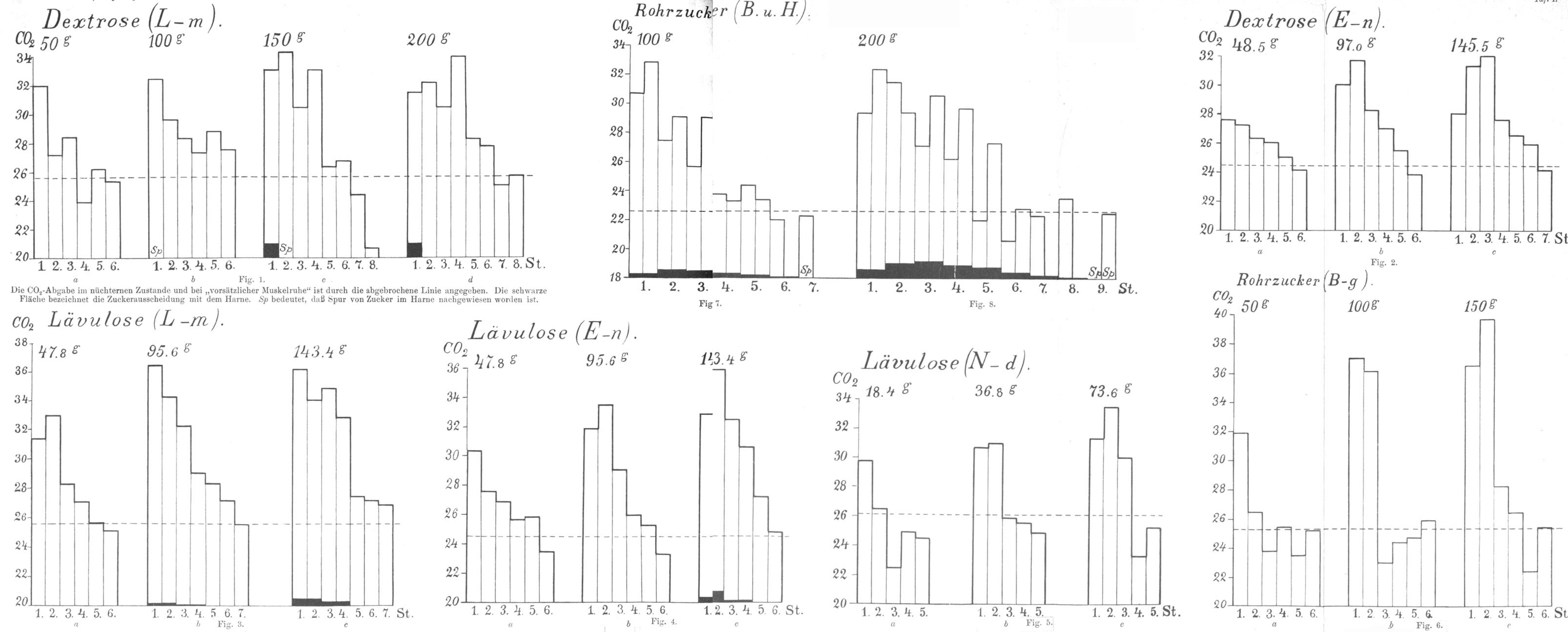


Dextrose: gewöhnlicher Nahrungs-Zustand $\mathrm{CO}_{2} 75^{\mathrm{g}}(\mathrm{W}-\mathrm{d}) \quad 75^{\mathrm{g}}(\mathrm{S}-\mathrm{st}) \quad 75^{\mathrm{g}}(\mathrm{L}-\mathrm{hl}) \quad 100^{\mathrm{g}}$ (B.u.H) $28-$

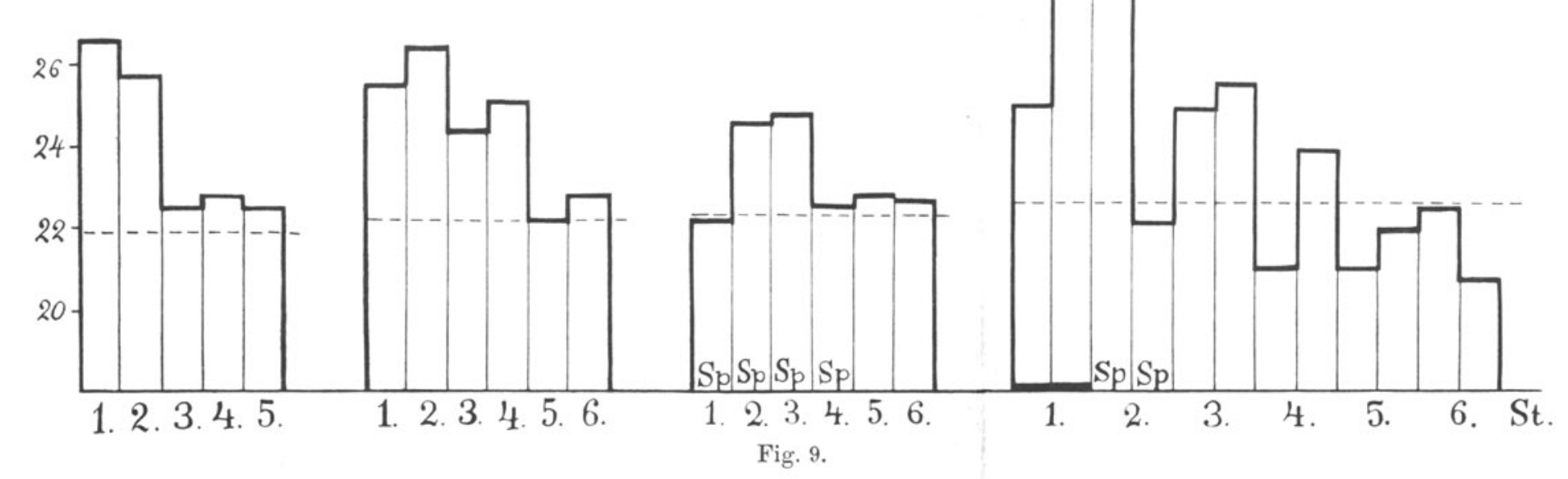

$\mathrm{CO}_{2}$ Lävulose: gewöhnlicher Nahrungs-Zustand

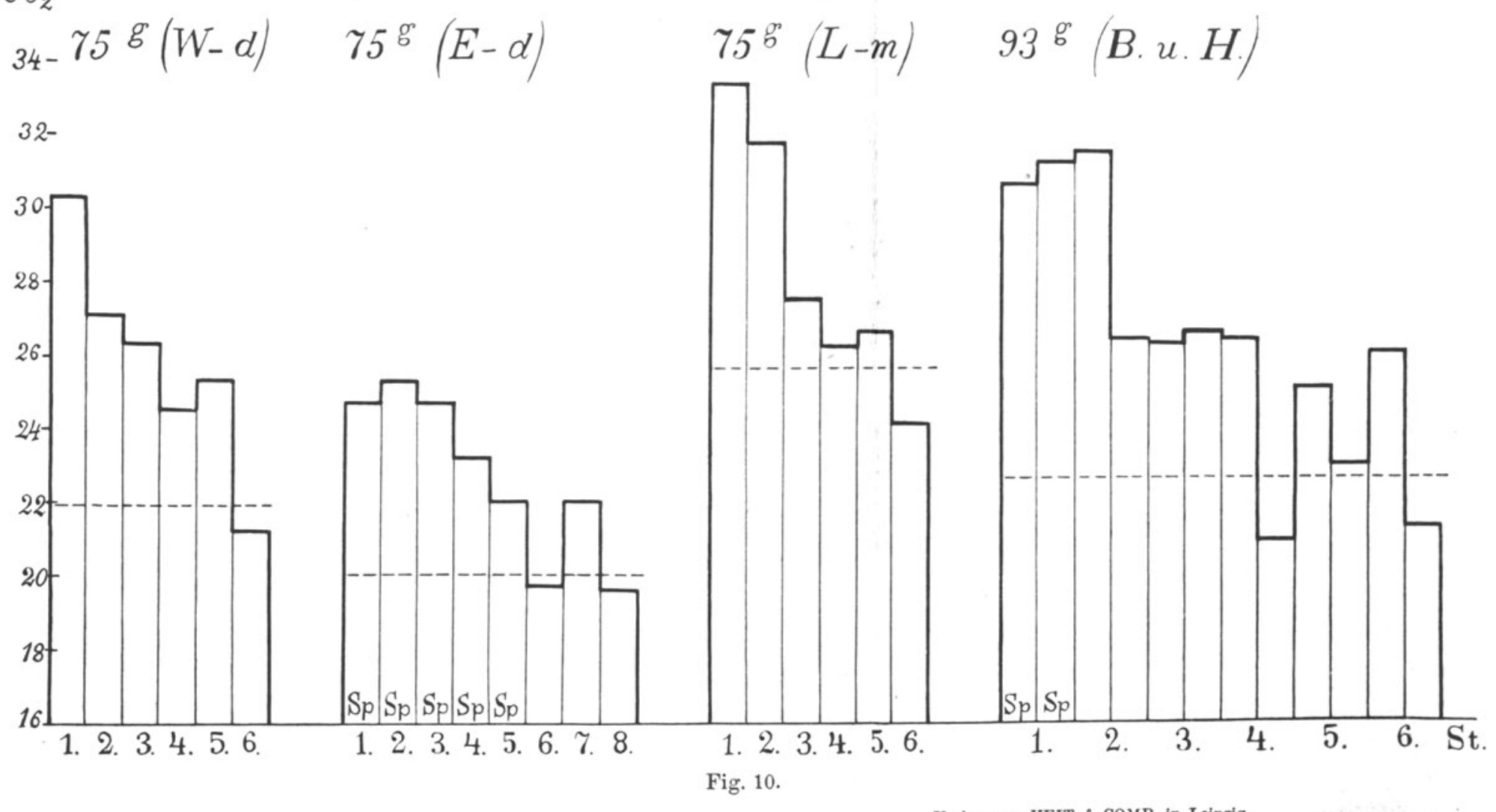

nach Glykogenverlust $\mathrm{CO}_{2}$$$
75^{g}(S-s t) \quad 75^{g}(L-h l)
$$

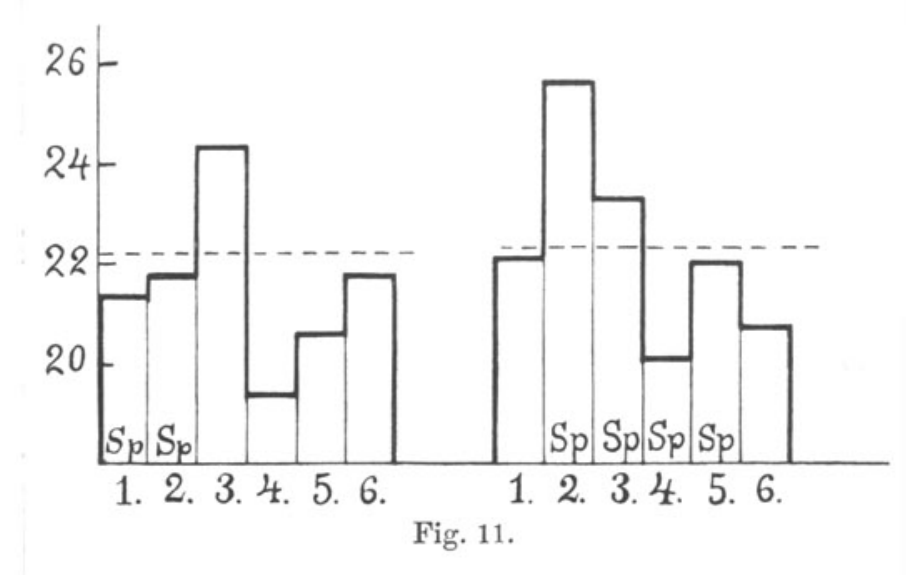

nach Glykogenverlust

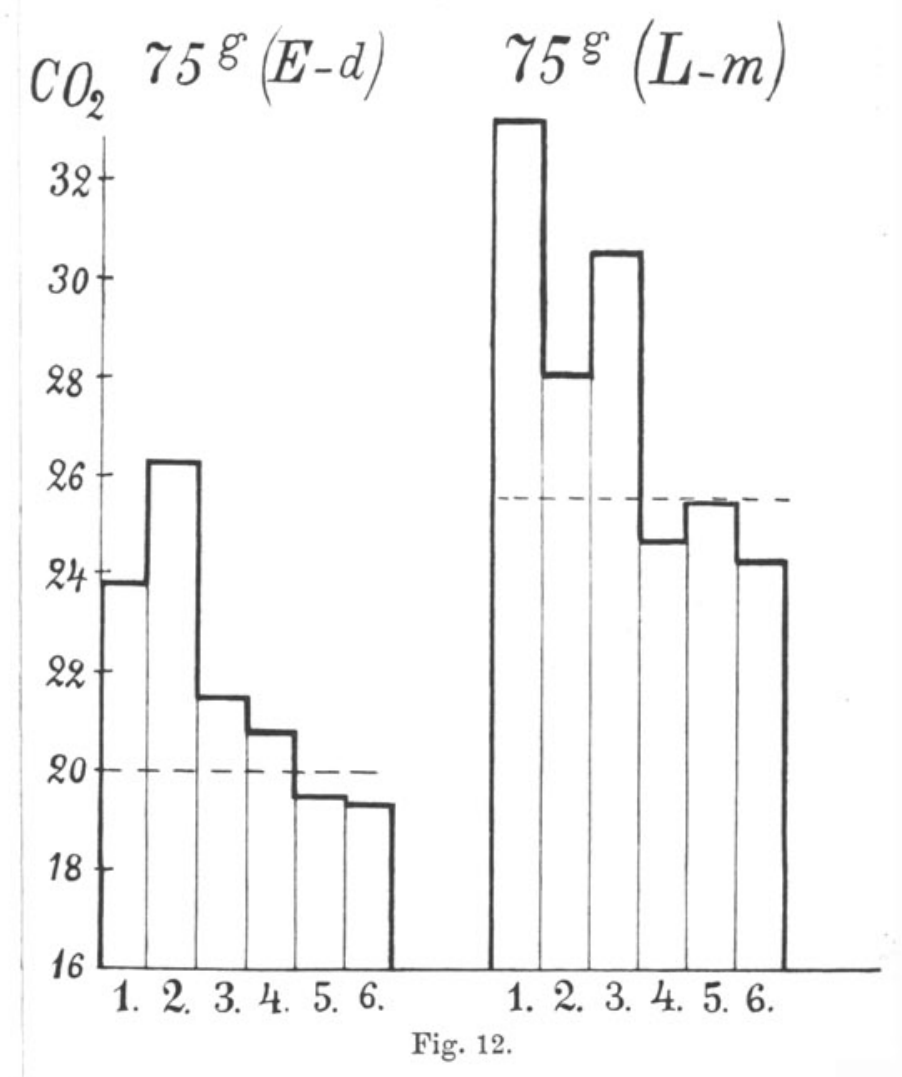


Galactose

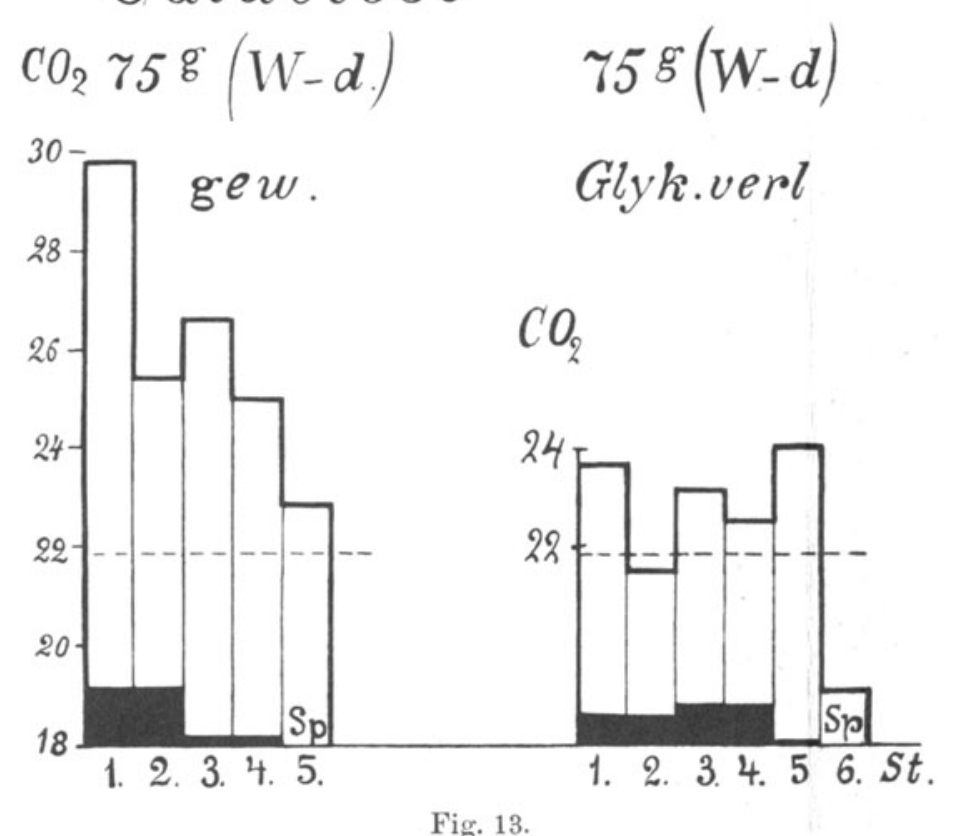

Rohrzucker

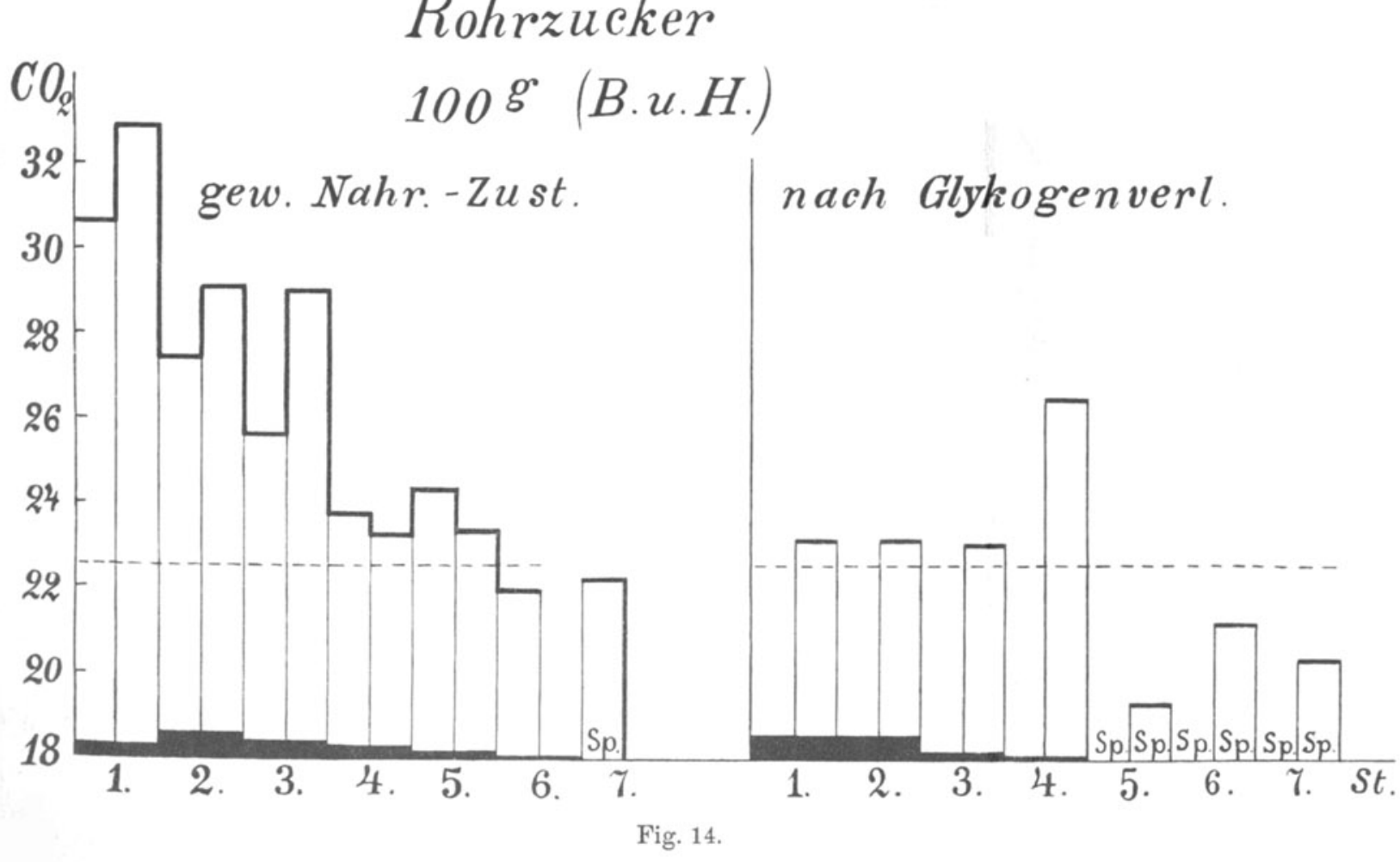

Dextrose

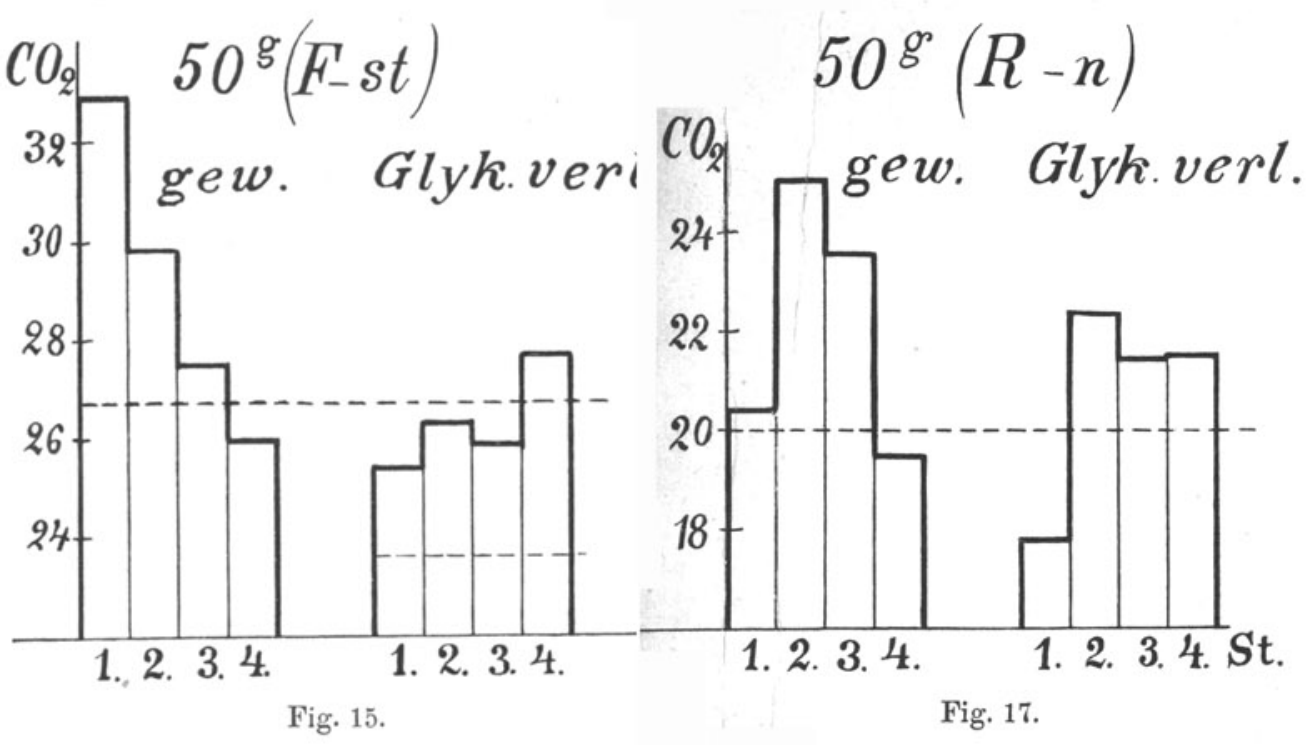

\section{Lävulose}

$\mathrm{CO}_{2} \quad 50^{\mathrm{g}}\left(\mathrm{F}_{-\mathrm{st}}\right) \quad \mathrm{CO}_{2} \quad 50^{\mathrm{g}}(\mathrm{R}-\mathrm{n})$

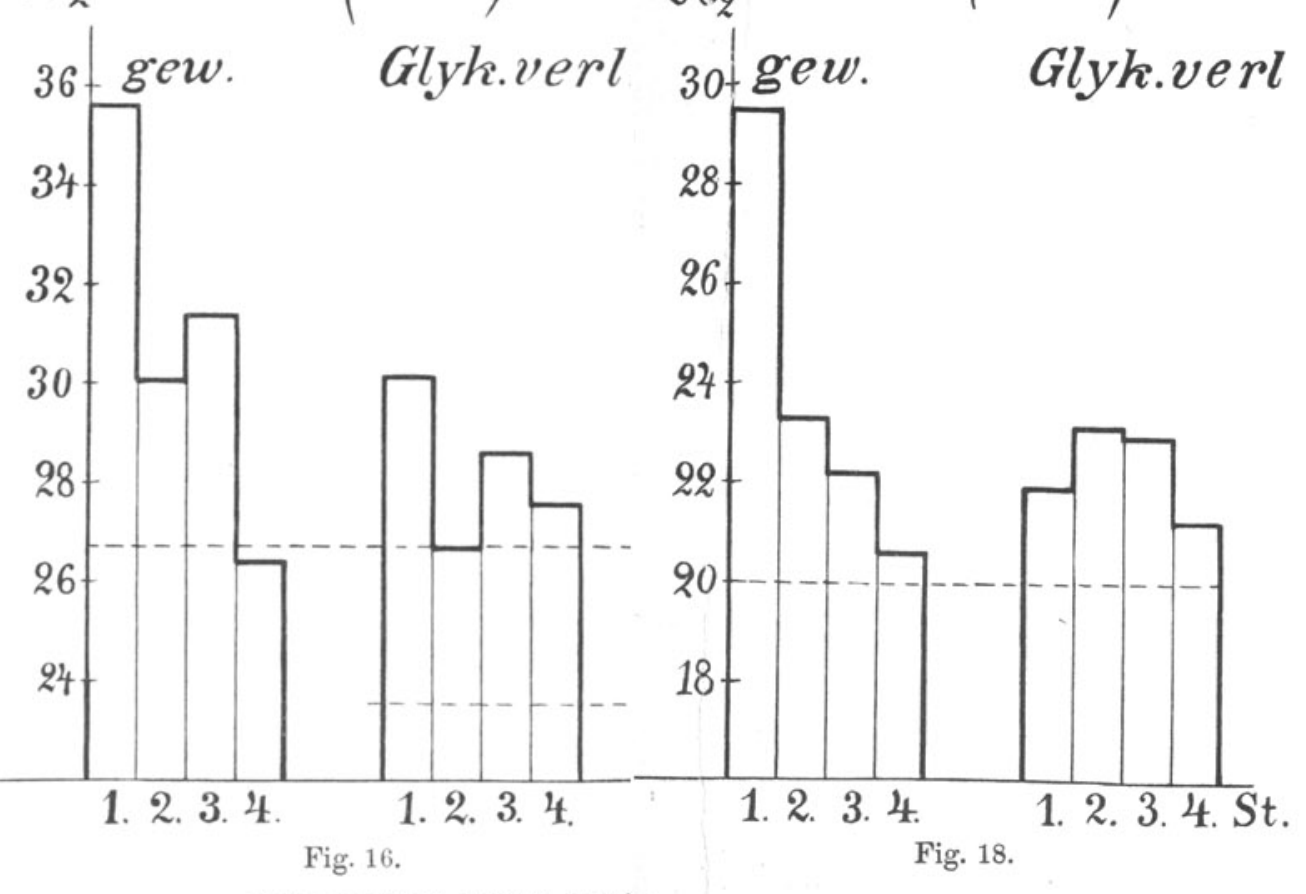

E-k-n. Diabet.

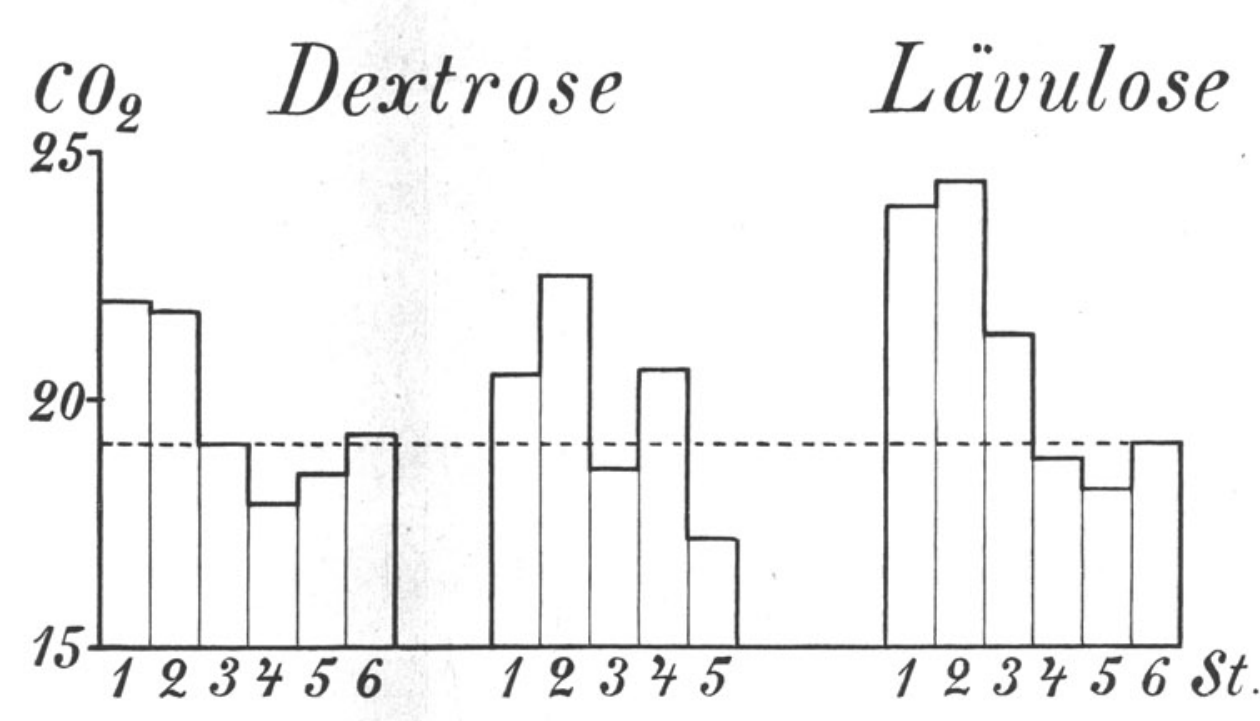
Zucker im Harn. Gm.

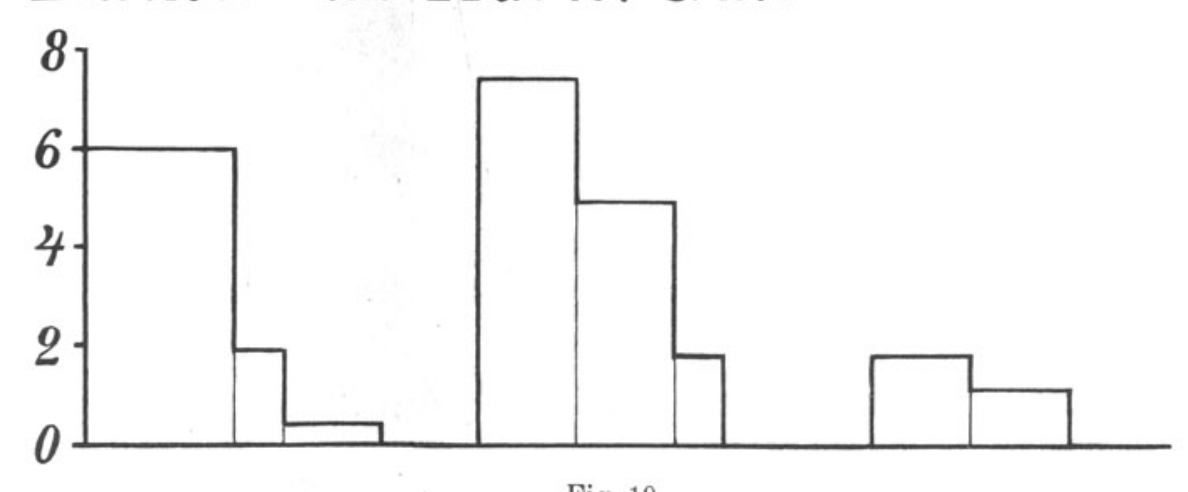


1.

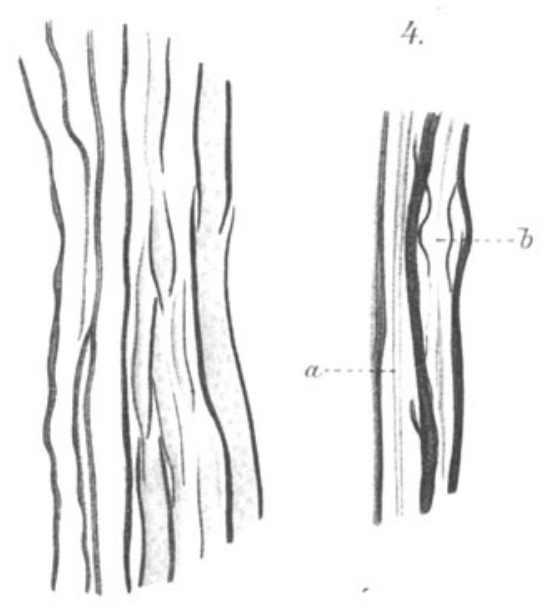

3.
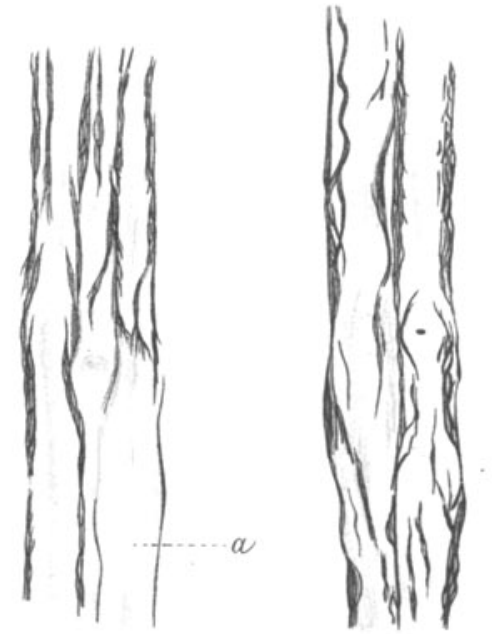
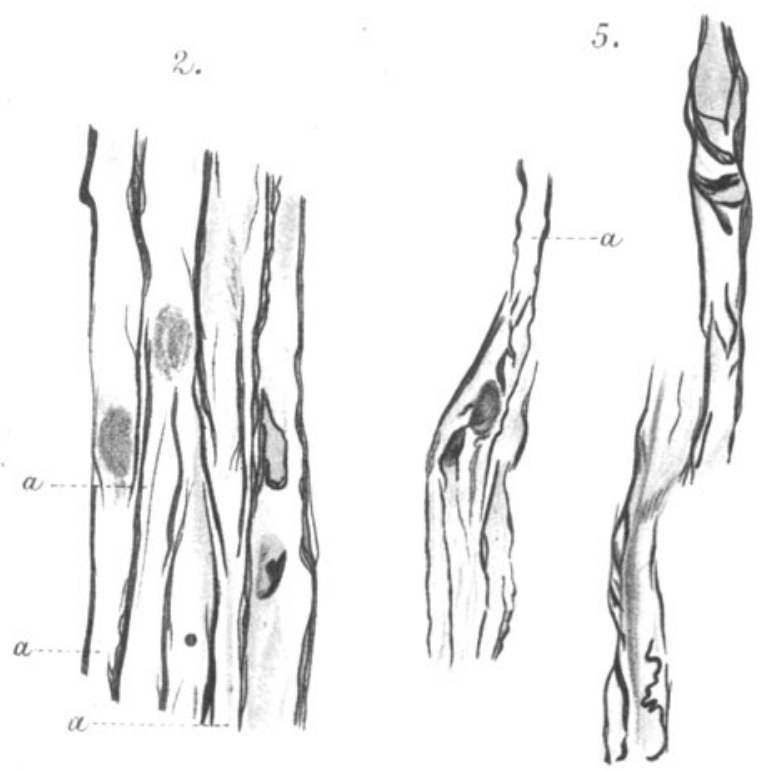

6.

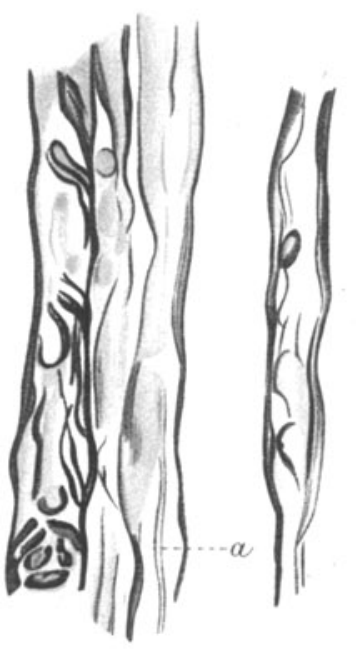




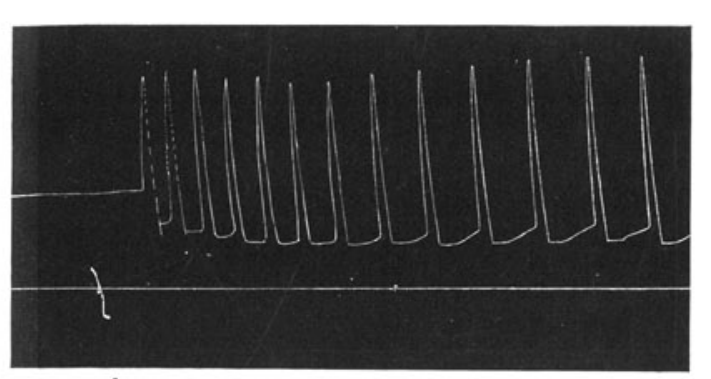

Fig. 1 a.

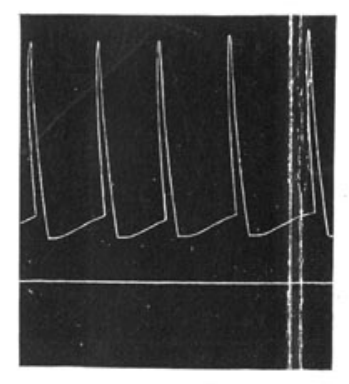

Fig. 1 b.

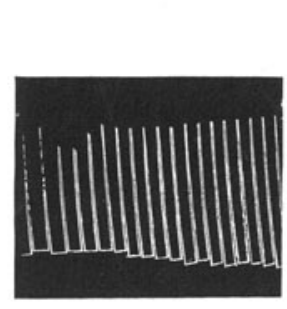

Fig. $3 b$.

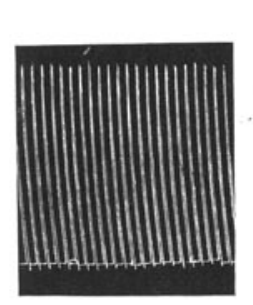

Fig. $3 \mathrm{c}$.

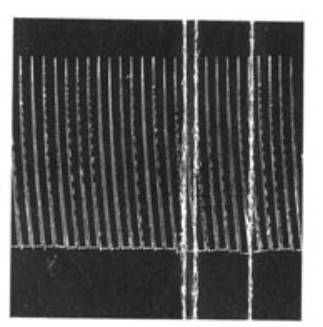

Fig. 3d.

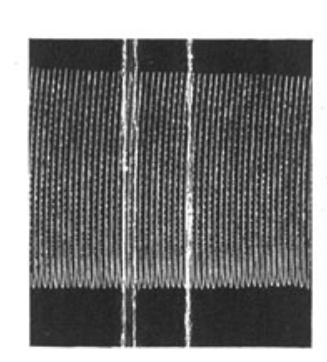

Fig. 4:b.

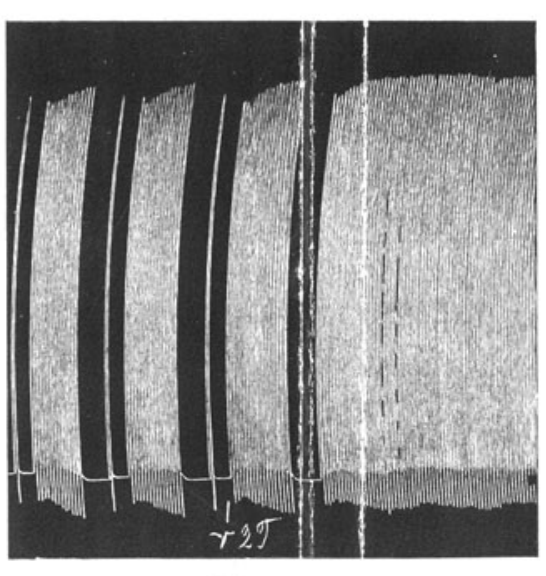

Fig. 5 b.

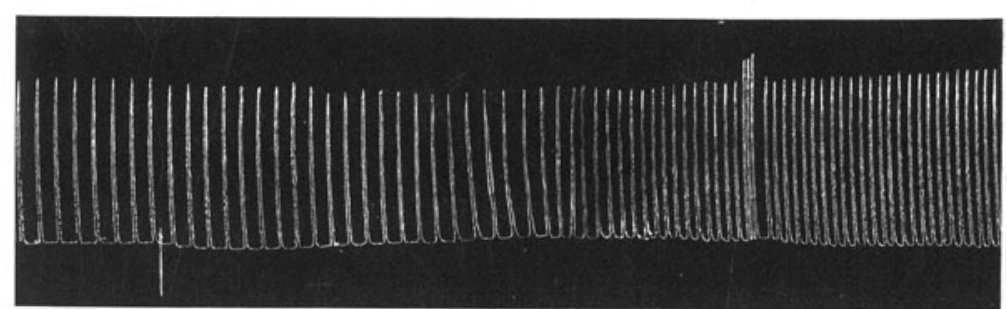

Fig. 9 a.

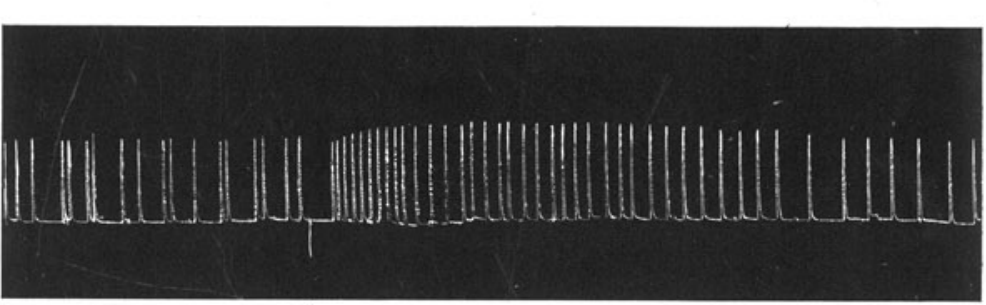

Fig. 6a.

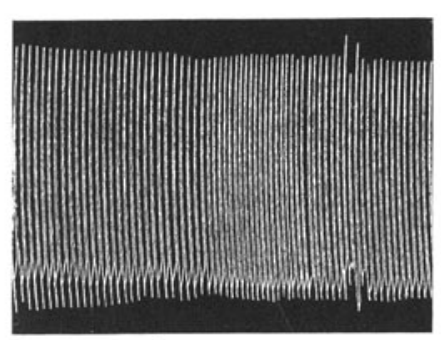

Fig. 7a.

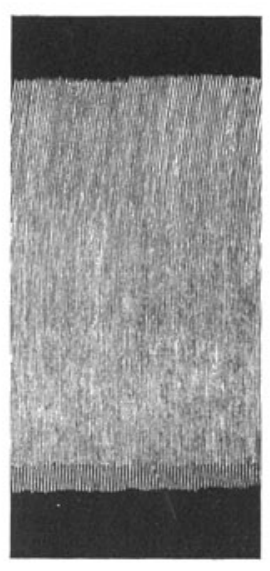

Fig. ฮ̃ c.

Fig. 7 b.

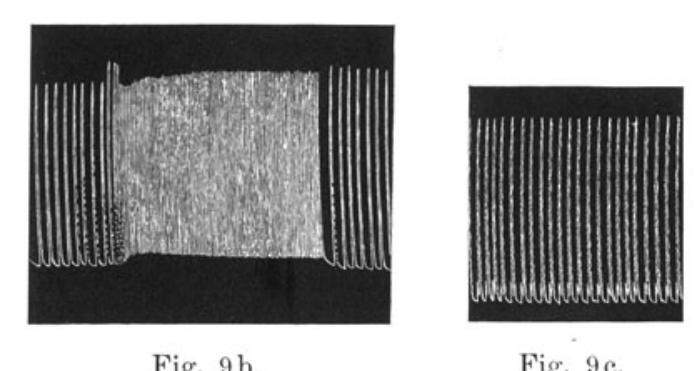

Fig. $9 \mathrm{~b}$.

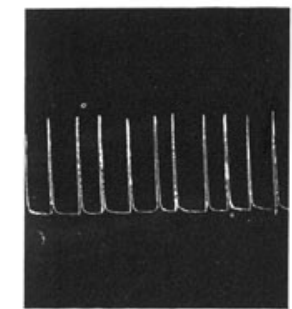

Fig. 6 b.

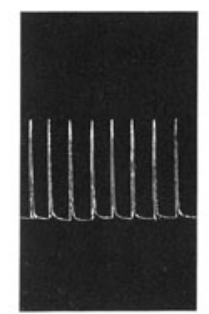

Fig. 6e.

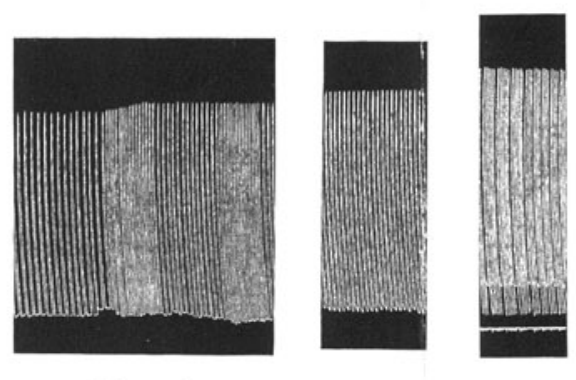

Fig. 7 c. Fig. 7 d.

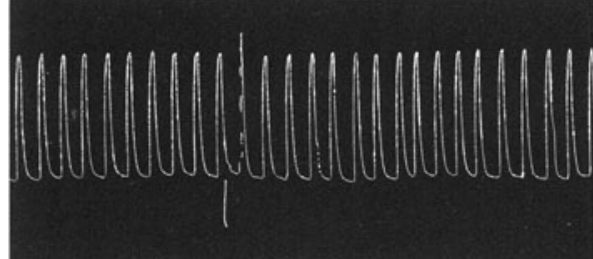

Fig. 8a.

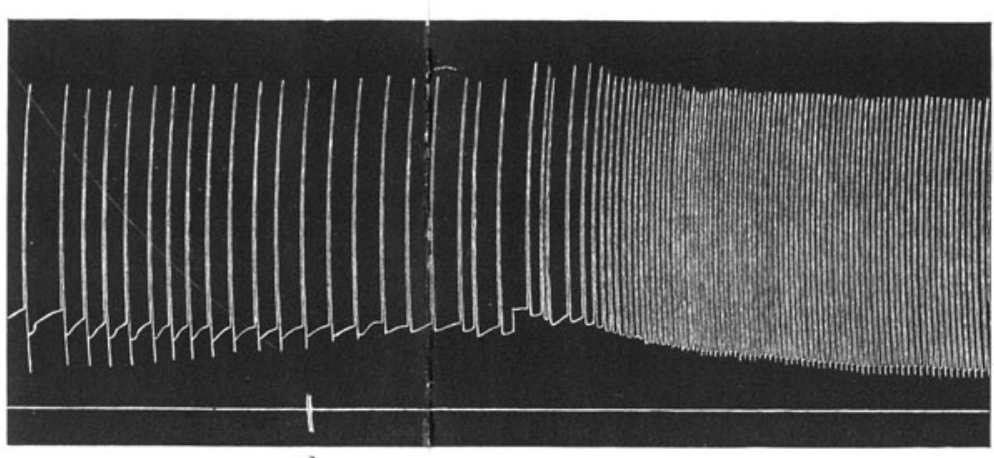

Fig. 2a.

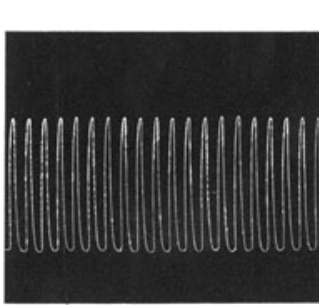

Fig. $8 \mathrm{~b}$.

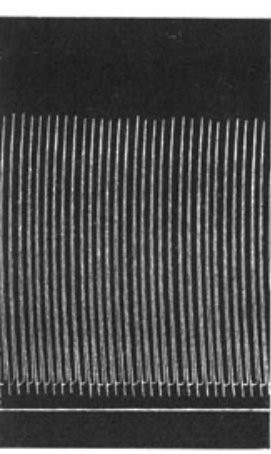

Fig. $2 b$. 


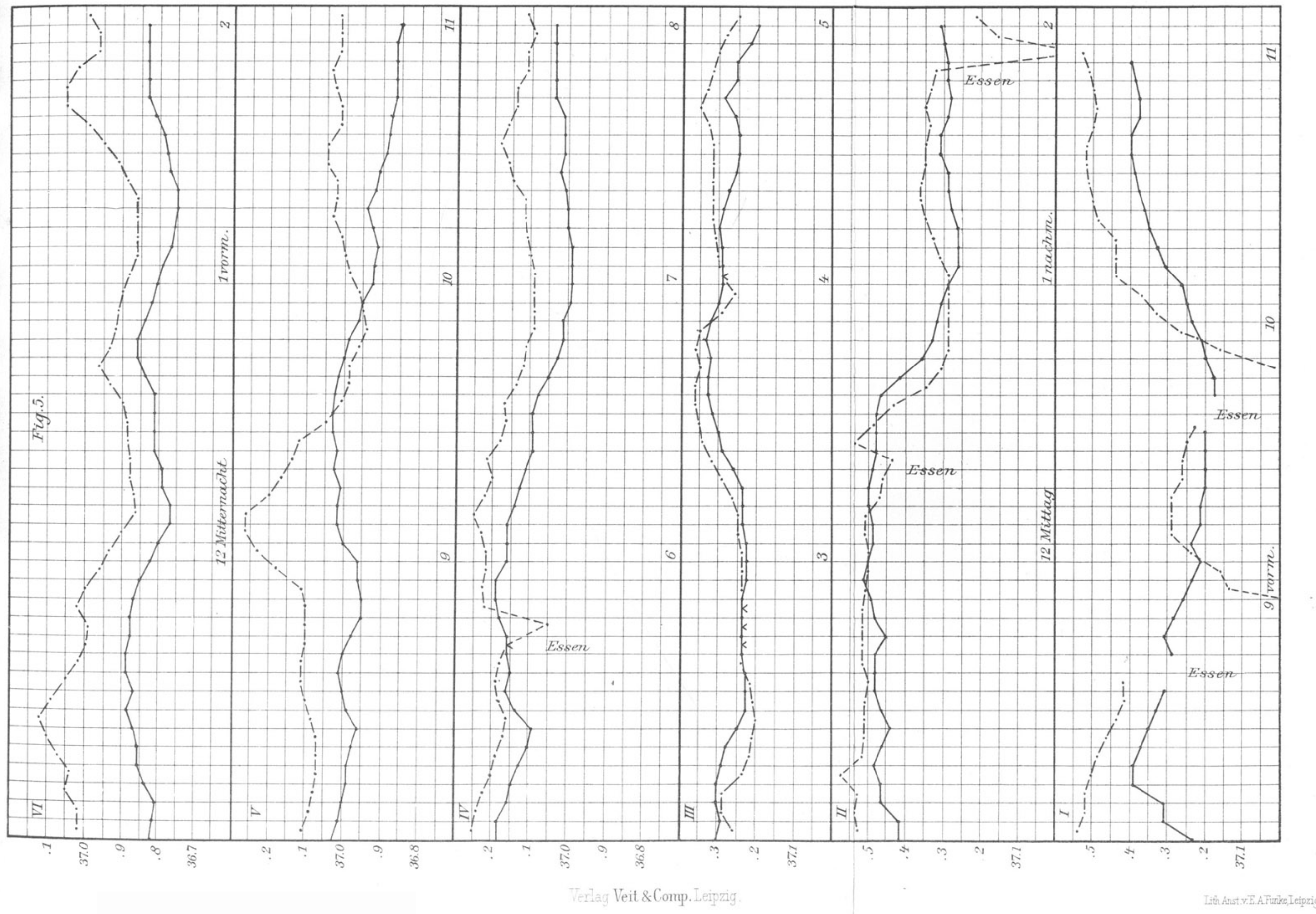


Skandin. Arch.f.Physiol. Bd. XXI.
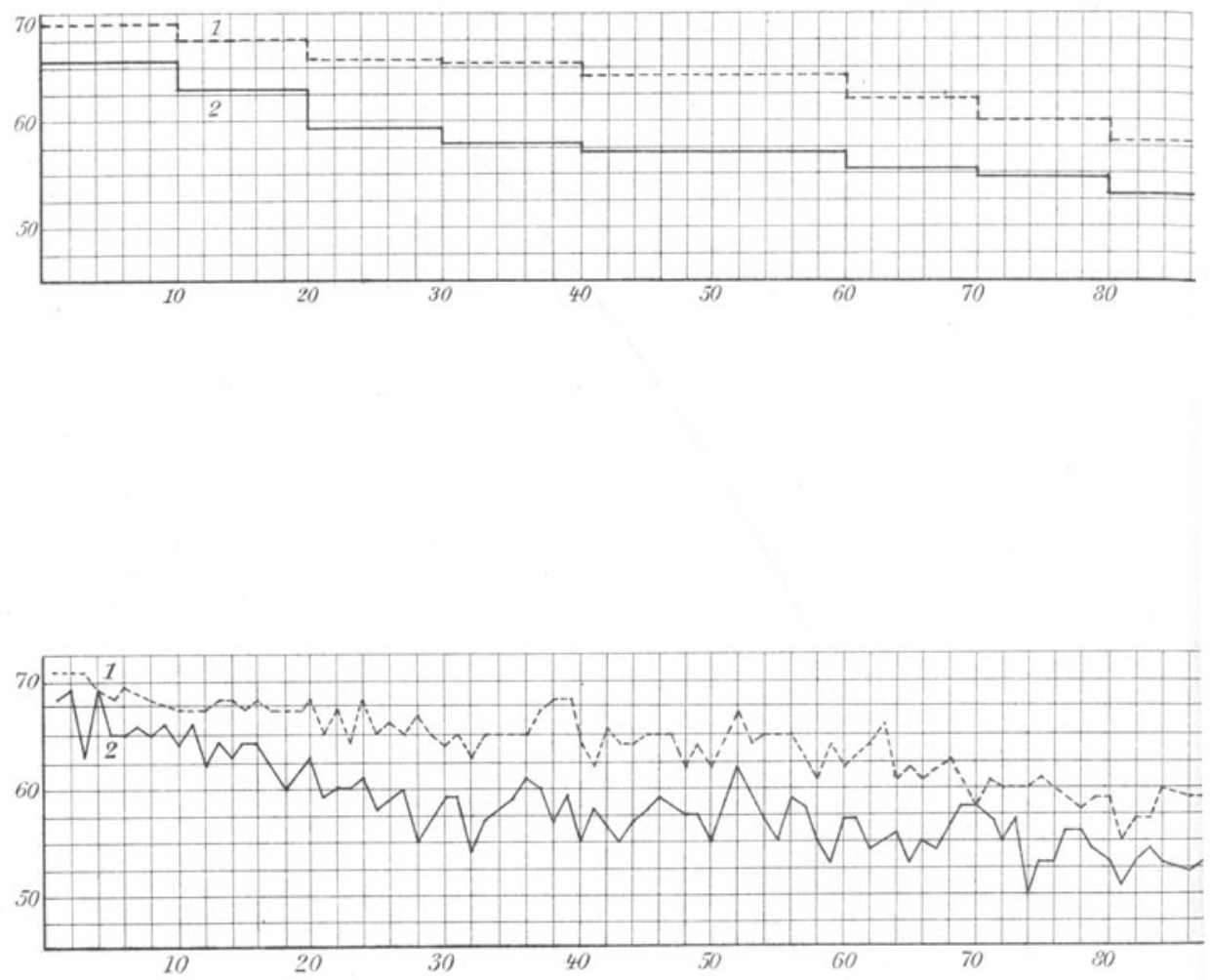

Fig.4.

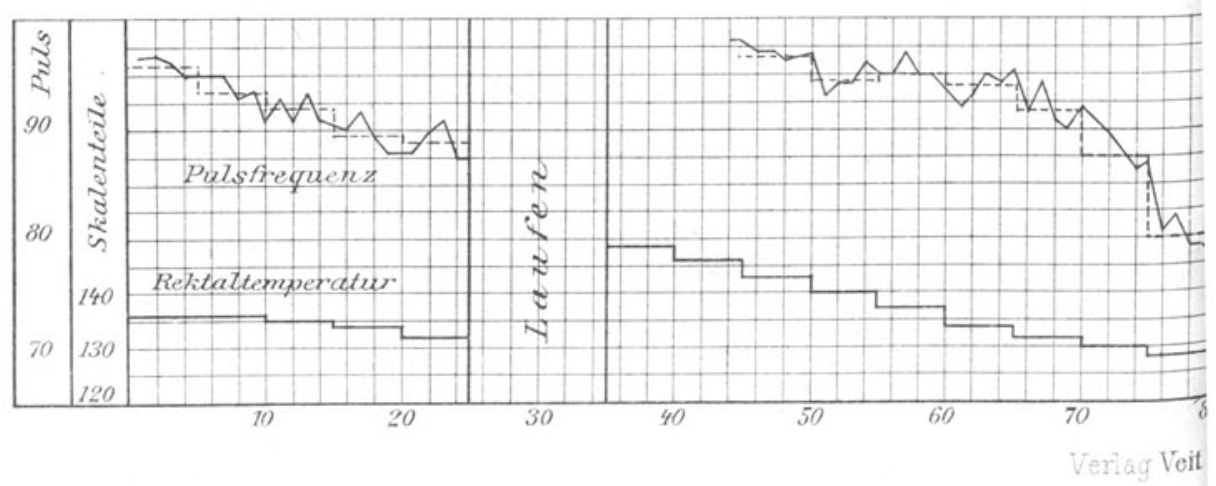


Fig.1.

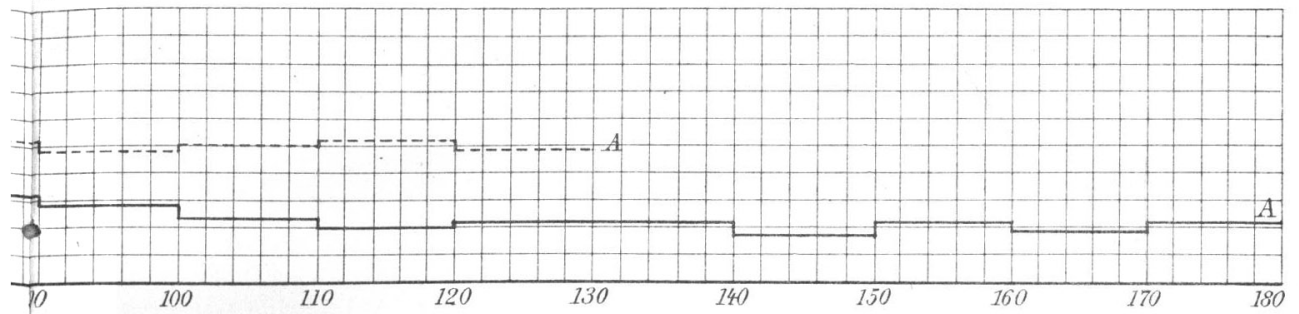

H.g. 2 .

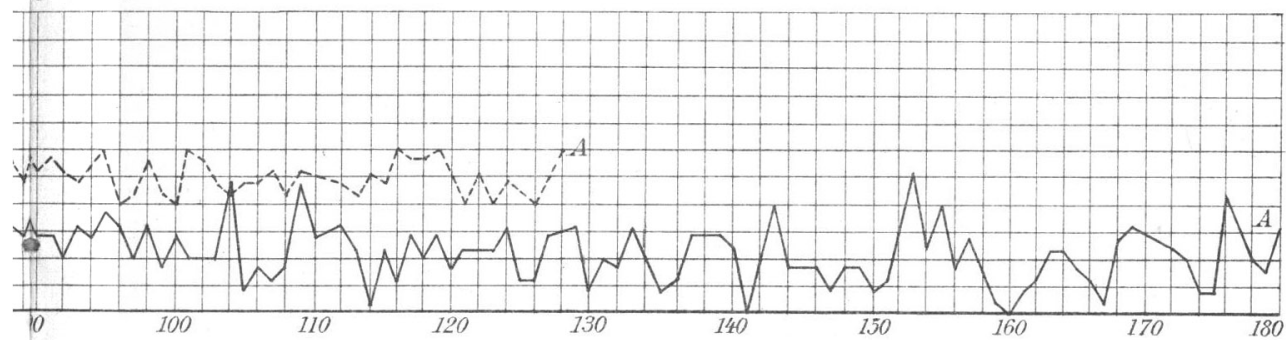

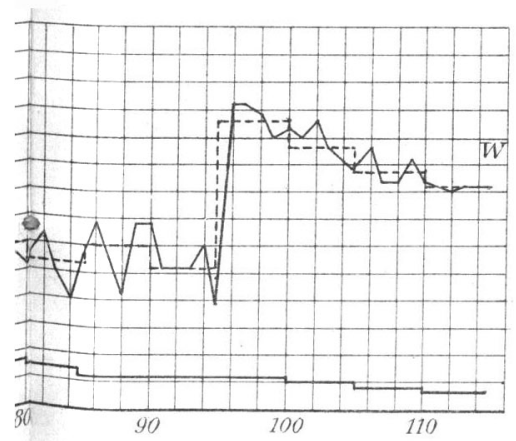

\&Comp. Leinzig.
Fig.5.

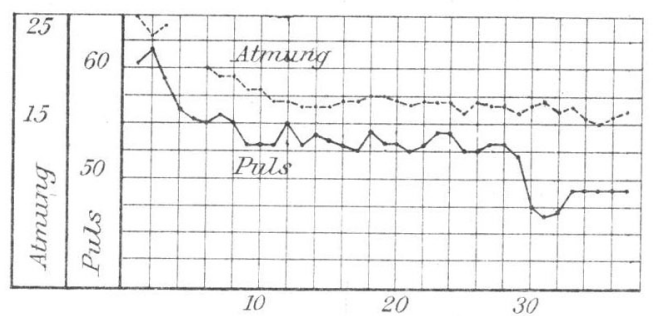

Inih Anstw E.A.tunke, I gipzig. 